\title{
Optimization of Single-Dose VSV-Based COVID-19 Vaccination in Hamsters
}

\author{
Kyle L. O'Donnell ${ }^{1}$, Chad S. Clancy ${ }^{2}$, Amanda J. Griffin ${ }^{1}$, Kyle Shifflett ${ }^{1}$, \\ Tylisha Gourdine ${ }^{1}$, Tina Thomas ${ }^{2}$, Carrie M. Long ${ }^{3}$, Wakako Furuyama ${ }^{1}$ \\ and Andrea Marzi ${ }^{1 *}$ \\ ${ }^{1}$ Laboratory of Virology, Division of Intramural Research, National Institute of Allergy and Infectious Diseases, National \\ Institutes of Health, Hamilton, MT, United States, ${ }^{2}$ Rocky Mountain Veterinary Branch, Division of Intramural Research, \\ National Institute of Allergy and Infectious Diseases, National Institutes of Health, Hamilton, MT, United States, ${ }^{3}$ Laboratory \\ of Bacteriology, Division of Intramural Research, National Institute of Allergy and Infectious Diseases, National Institutes of \\ Health, Hamilton, MT, United States
}

OPEN ACCESS

Edited by: Fabio Bagnoli,

GlaxoSmithKline, Italy

Reviewed by: Catalina Florez,

United States Army Medical Research Institute of Infectious Diseases (USAMRIID), United States Vladimir Alexeevich Gushchin,

N.F. Gamaleya Scientific Research Institute of Epidemiology and Microbiology (RAMS), Russia

*Correspondence: Andrea Marzi marzia@niaid.nih.gov

Specialty section:

This article was submitted to Vaccines and Molecular Therapeutics, a section of the journal

Frontiers in Immunology

Received: 01 October 2021 Accepted: 14 December 2021 Published: 06 January 2022

Citation:

O'Donnell KL, Clancy CS, Griffin AJ, Shifflett K, Gourdine T, Thomas T, Long CM, Furuyama W and Marzi A (2022) Optimization of Single-Dose VSV-Based COVID-19 Vaccination in Hamsters.

Front. Immunol. 12:788235. doi: 10.3389/fimmu.2021.788235
The ongoing COVID-19 pandemic has resulted in global effects on human health, economic stability, and social norms. The emergence of viral variants raises concerns about the efficacy of existing vaccines and highlights the continued need for the development of efficient, fast-acting, and cost-effective vaccines. Here, we demonstrate the immunogenicity and protective efficacy of two vesicular stomatitis virus (VSV)-based vaccines encoding the SARS-CoV-2 spike protein either alone (VSV-SARS2) or in combination with the Ebola virus glycoprotein (VSV-SARS2-EBOV). Intranasally vaccinated hamsters showed an early $\mathrm{CD} 8^{+} \mathrm{T}$ cell response in the lungs and a greater antigen-specific lgG response, while intramuscularly vaccinated hamsters had an early $\mathrm{CD}^{+} \mathrm{T}$ cell and NK cell response. Intranasal vaccination resulted in protection within 10 days with hamsters not showing clinical signs of pneumonia when challenged with three different SARS-CoV-2 variants. This data demonstrates that VSV-based vaccines are viable single-dose, fast-acting vaccine candidates that are protective from COVID-19.

Keywords: severe acute respiratory syndrome coronavirus-2, SARS-CoV-2, vesicular stomatitis virus, VSV-SARS2, VSV-EBOV, rVSV-ZEBOV GP

\section{INTRODUCTION}

Severe acute respiratory syndrome coronavirus-2 (SARS-CoV-2) has emerged as a novel, highly infectious, respiratory CoV and is the causative agent of Coronavirus disease 2019 (COVID-19), first described in the city of Wuhan in Hubei province in China (1). The World Health Organization declared the SARS-CoV-2 pandemic a Public Health Emergency of International Concern on January $30^{\text {th }} 2020$ (2). Clinically, COVID-19 can lead to respiratory distress and, in some cases, respiratory failure (3). CoVs are enveloped, single-stranded positive-sense RNA viruses with a $30 \mathrm{~kb}$ genome and 5 open reading frames including the four major structural proteins: spike (S), envelope, membrane, and nucleocapsid (N). The S mediates binding of SARS-CoV-1 and SARS-CoV-2 to angiotensin-converting enzyme 2 (ACE2) on the surface of various cell types including epithelial cells of the respiratory tract (4-6). The COVID-19 pandemic mandated the development of a 
vaccine to be a global priority $(7-11)$. Due to the mutagenic nature of the replication of RNA viruses, new viral variants of concern (VOC) have emerged to dominate the pathogenic landscape. Two of the first variants that emerged were B.1.1.7 (UK; alpha variant) and B.1.351 (South Africa, SA; beta variant). B.1.1.7 acquired 23 mutations including N501Y within the $S$ shown to increase binding affinity to the ACE2 receptor $(12,13)$. B.1.351 harbors similar mutations such as the N501Y, in addition to $\mathrm{K} 417 \mathrm{~N}$ and $\mathrm{E} 484 \mathrm{~K}$ which may reduce the efficacy of existing countermeasures (14-16).

An ideal vaccine candidate would be safe, effective, rapidly deployable, require only a single immunization, and retain efficacy against multiple variants. Currently, vaccine candidates express the trimeric SARS-CoV-2 S as the primary antigen. One mRNA-based vaccine and an adenovirus-based vector have received emergency use authorization by the Food and Drug Administration (FDA) in the United States, and another mRNA vaccine recently received full FDA approval (17). All utilize the S as the primary antigen and elicit $\mathrm{T}$ cell and antigen-specific IgG responses (18-20). The route of vaccination can greatly influence the local immune environment at the site of vaccination. A study comparing intramuscular (IM) and intranasal (IN) vaccination of mice with a chimpanzee adenoviral vector-based COVID-19 vaccine revealed an increase in stimulation of local mucosal immunity. Local mucosal immunity was improved after IN vaccination demonstrated by antigen specific IgA and lung resident $\mathrm{T}$ cell generation (21). Benefits of IN vaccination have been demonstrated for other adenoviral vector vaccines as well as subunit vaccines, which lead to the exploration of optimal route of vaccination in this study (22-24).

The recombinant vesicular stomatitis virus (VSV) vaccine platform has previously been used for multiple viral pathogens such as Ebola, Nipah, and Lassa (25-27). We developed two VSV-based vaccines for SARS-CoV-2: a monovalent and a bivalent vaccine construct. The monovalent construct expresses the S of SARS-CoV-2 (VSV-SARS2) with a cytoplasmic tail deletion, which has been previously described $(28,29)$. Recently, a similar VSV-based vaccine expressing the fulllength $S$ demonstrated protective efficacy against COVID-19 in Syrian golden hamsters challenged 23 days after IM vaccination (30). The bivalent vaccine co-expresses the full-length $S$ and the Ebola virus (EBOV) GP (VSV-SARS2-EBOV) with the EBOV GP facilitating virus recovery and expanding target cell range. The VSV vaccine platform displays several advantages to other similar approaches. VSV-based vaccines have been shown to produce a robust and rapid immune response to the encoded antigen(s) after a single immunization. Other viral vector vaccines have the problem of preexisting immunity; with VSV preexisting immunity would be directed primarily against the glycoprotein, which is not present in this system (31). The time to immunity has been demonstrated to be 7 to 10 days for a number of pathogens, greatly reducing the time needed between vaccination and protection $(31,32)$. Multiple routes of vaccination have been shown to be efficacious utilizing VSVbased vaccines, such as IM and IN (31-35). Previously, we determined the efficacy of IM and IN vaccination of nonhuman primates (NHP) with VSV-SARS2-EBOV. The study demonstrated that IM vaccination resulted in superior protective efficacy with a short time to challenge, however, IN vaccination might be similar with a longer time between vaccination and challenge (36). These unique attributes robust immune stimulation and short time to immunity make VSV an attractive viral vector vaccine platform for SARS-CoV-2.

Syrian golden hamsters have previously been established as a model system for SARS-CoV-2 recapitulating respiratory disease $(37,38)$. When IN-challenged, these animals develop moderate broncho-interstitial pneumonia with peak viral replication in the lungs 3 days post challenge (DPC) resolving by day 10. Peak histopathologic lesions in the lungs have been observed between 3- to 5- DPC (38). In this Syrian golden hamster study, we sought to determine the humoral and cellular immunogenicity over time in response to two VSV-based SARS-CoV-2 vaccines through both IN- and IM-vaccination routes at two challenge timepoints. We show that both vaccines offer protective immunity against multiple viral variants in the Syrian golden hamster model.

\section{METHODS}

\section{Ethics Statement}

All infectious work with SARS-CoV-2 was performed in the high-containment laboratories at the Rocky Mountain Laboratories (RML), Division of Intramural Research, National Institute of Allergy and Infectious Diseases, National Institutes of Health. RML is an institution accredited by the Association for Assessment and Accreditation of Laboratory Animal Care International (AAALAC). All procedures followed standard operating procedures (SOPs) approved by the RML Institutional Biosafety Committee (IBC). Animal work was performed in strict accordance with the recommendations described in the Guide for the Care and Use of Laboratory Animals of the National Institute of Health, the Office of Animal Welfare and the Animal Welfare Act, United States Department of Agriculture. The studies were approved by the RML Animal Care and Use Committee. Procedures were conducted in animals anesthetized by trained personnel under the supervision of veterinary staff. All efforts were made to ameliorate animal welfare and minimize animal suffering; food and water were available ad libitum.

\section{Animal Study}

Two hundred and fifty Syrian golden hamsters (5-8 weeks of age; male and female) were used in this study. The hamsters were randomly selected into groups as shown in Table S1. On the day of vaccination hamsters received a single dose of $1 \times 10^{5}$ plaqueforming units (PFU) of VSV-SARS2-EBOV or VSV-SARS2 by the IM (thigh) or IN route. Control animals received the same dose of a control vaccine (VSV-EBOV) by either the IM or IN route. On days 3, 10, and 38 animals were euthanized for sample collection to analyze vaccine immunogenicity. For efficacy studies with 28 and 10 days between vaccination and challenge 
animals received the same vaccine dose by the routes mentioned above. All hamsters were challenged IN with $1 \times 10^{5}$ median tissue culture infectious dose $\left(\mathrm{TCID}_{50}\right)$ SARS-CoV-2 as previously described (38). On 4 DPC, all animals were euthanized for sample collection.

\section{Cells and Viruses}

Huh7 and VeroE6 cells were grown at $37^{\circ} \mathrm{C}$ and $5 \% \mathrm{CO}_{2}$ in Dulbecco's modified Eagle's medium (DMEM) (Sigma-Aldrich, St. Louis, MO) containing 10\% fetal bovine serum (FBS) (Wisent Inc., St. Bruno, Canada), $2 \mathrm{mM}$ L-glutamine (Thermo Fisher Scientific, Waltham, MA), $50 \mathrm{U} / \mathrm{mL}$ penicillin (Thermo Fisher Scientific), and $50 \mu \mathrm{g} / \mathrm{mL}$ streptomycin (Thermo Fisher Scientific). BHK-T7 (baby hamster kidney) cells expressing the $\mathrm{T} 7$ polymerase were grown at $37^{\circ} \mathrm{C}$ and $5 \% \mathrm{CO}_{2}$ in minimum essential medium (39) (Thermo Fisher Scientific) containing 10\% tryptose phosphate broth (Thermo Fisher Scientific), 5\% FBS, 2 mM L-glutamine, 50 $\mathrm{U} / \mathrm{mL}$ penicillin, and $50 \mu \mathrm{g} / \mathrm{mL}$ streptomycin. Ancestral SARSCoV-2 isolate nCoV-WA1-2020 (MN985325.1) (40), SARS-CoV-2 isolate B.1.1.7 (hCOV_19/England/204820464/2020), SARS-CoV-2 isolate B.1.351 (hCoV-19/South African/KRISP-K005325/2020) or SARS-CoV-2 isolate B.1.617.2 (hCoV-19/USA/KY-CDC-24242084/2021) were used in these studies. The following reagent was obtained through BEI Resources, NIAID, NIH: Severe Acute Respiratory Syndrome-Related Coronavirus 2, Isolate hCoV-19/ England/204820464/20200, NR-54000, contributed by Bassam Hallis. SARS-CoV-2 B. 1.351 was obtained with contributions from Dr. Tulio de Oliveira and Dr. Alex Sigal (Nelson R Mandela School of Medicine, UKZN). SARS-CoV-2 B.1.617.2 was obtained with contributions from B. Zhou, N. Thornburg, and S. Tong (Centers for Disease Control and Prevention, USA). All viruses were grown and titered on Vero E6 cells, and sequence confirmed.

\section{Generation of VSV-Based Vaccine Candidates}

The SARS-CoV-2 S open reading frame was PCR-amplified from an expression plasmid encoding the codon-optimized (human) gene based on GenBank accession number MN908947 which was kindly provided by Vincent Munster (NIAID). Full-length SARS-CoV-2 $S$ was cloned into the pATX-VSV-EBOV plasmid upstream of the EBOV-Kikwit GP resulting in VSV-SARS2EBOV (Figure S1A) following a previously successful strategy (41). The cytoplasmic tail deletion was introduced by PCR and was cloned into the pATX-VSV plasmid resulting in VSVSARS2. The replication competent recombinant VSV was recovered in BHK-T7 cells as described previously (42). VSVSARS2-EBOV was propagated in Huh7 cells. The complete sequence of the virus was confirmed by Sanger sequencing. The titer of the virus stock was quantified using standard plaque assay on VeroE6 cells.

\section{Growth Kinetics}

VeroE6 cells were grown to confluency in a 12-well plate and infected in triplicate with VSVwt, VSV-EBOV, VSV-SARS2, or VSV-SARS2-EBOV at a multiplicity of infection of 0.01 . After 1 $\mathrm{h}$ incubation at $37^{\circ} \mathrm{C}$, cells were washed three times with plain DMEM, and covered with DMEM containing 2\% FBS.
Supernatant samples were collected at $0,6,12,24,48,72$, and 96 hours post infection and stored at $-80^{\circ} \mathrm{C}$. The titer of the supernatant samples was determined performing $\mathrm{TCID}_{50}$ assay on VeroE6 cells as previously described (42).

\section{Western Blot Analysis}

Supernatant samples containing VSV were mixed 1:1 with sodium dodecyl sulfate-polyacrylamide (SDS) gel electrophoresis sample buffer containing $20 \% \beta$-mercaptoethanol and heated to $99^{\circ} \mathrm{C}$ for $10 \mathrm{~min}$. SDS-PAGE and transfer to Trans-Blot polyvinylidene difluoride membranes (Bio-Rad Laboratories) of all samples was performed as described elsewhere (34). Protein detection was performed using anti-SARS-CoV-2 S RBD (1:1000; Sino Biological) or anti-EBOV GP (ZGP 12/1.1, $1 \mu \mathrm{g} / \mathrm{ml}$; kindly provided by Ayato Takada, Hokkaido University, Japan) or antiVSV M (23H12, 1:1000; Kerafast Inc.). After horse-radish peroxidase (HRP)-labeled secondary antibody staining using either anti-mouse IgG $(1: 10,000)$ or anti-rabbit IgG (1:5000) (Jackson ImmunoResearch), the blots were imaged using the SuperSignal West Pico chemiluminescent substrate (Thermo Fisher Scientific) and an iBright ${ }^{\mathrm{TM}}$ CL1500 Imaging System (Thermo Fisher Scientific).

\section{RNA Extraction and RT-qPCR}

Nasal swab samples were extracted using the QIAamp Viral RNA Mini Kit (QIAGEN) according to manufacturer specifications. Tissues, a maximum of $30 \mathrm{mg}$ each, were processed and extracted using the RNeasy Mini Kit (QIAGEN) according to manufacturer specifications. One step RT-qPCR for genomic viral RNA was performed using specific primer-probe sets and the QuantiFast Probe RT-PCR +ROX Vial Kit (QIAGEN), in the Rotor-Gene Q (QIAGEN) as described previously (43). Five $\mu \mathrm{L}$ of each RNA extract were run alongside dilutions of SARS-CoV-2 standards with a known concentration of RNA copies.

\section{Enzyme-Linked Immunosorbent Assay}

Serum samples from SARS-CoV-2 infected animals were inactivated by $\gamma$-irradiation and used in BSL2 according to IBC-approved SOPs. NUNC Maxisorp Immuno plates were coated with $50 \mu \mathrm{l}$ of $1 \mu \mathrm{g} / \mathrm{mL}$ of recombinant SARS-CoV-2 S $(\mathrm{S} 1+\mathrm{S} 2)$, SARS-CoV-2 RBD (Sino Biological) or EBOV GP (32) at $4^{\circ} \mathrm{C}$ overnight and then washed three times with PBS containing $0.05 \%$ Tween 20 (PBST). The plates were blocked with 3\% skim milk in PBS for 3 hours at room temperature, followed by three additional washes with PBST. The plates were incubated with $50 \mu \mathrm{l}$ of serial dilutions of the samples in PBS containing $1 \%$ skim milk for 1 hour at room temperature. After three washes with PBST, the bound antibodies were labeled using $50 \mu \mathrm{l}$ of 1:2,500 peroxidase anti-hamster IgG $(\mathrm{H}+\mathrm{L})$ (SeraCare Life Sciences) diluted in 1\% skim milk in PBST. After incubation for $1 \mathrm{~h}$ at room temperature and three washes with PBST, $50 \mu \mathrm{l}$ of KPL ABTS peroxidase substrate solution mix (SeraCare Life Sciences) was added to each well, and the mixture was incubated for $30 \mathrm{~min}$ at room temperature. The optical density (OD) at 405 $\mathrm{nm}$ was measured using a GloMax ${ }^{\circledR}$ explorer (Promega) plate reader. The OD values were normalized to the baseline samples 
obtained with naïve hamster serum and the cutoff value was set as the mean OD plus standard deviation of the blank.

\section{Flow Cytometry}

Hamster peripheral blood mononuclear cells (PBMCs) were isolated from ethylene diamine tetraceticacid (EDTA) whole blood by overlay on a Histopaque ${ }^{\circledR}-1077$ density cushion and separated according to manufacturer's instructions. Tissues were processed into single cell suspensions as described previously (44). Cells were stimulated for 6 hours with media alone, cell stimulation cocktail (containing PMA-Ionomycin, Biolegend), $1 \mu \mathrm{g} / \mathrm{ml}$ SARS-CoV-2 S peptide pool (IDT), or Lassa virus (LASV) GPC peptide pool (IDT) together with $5 \mu \mathrm{g} / \mathrm{ml}$ Brefeldin A (Biolegend). Following surface staining with Live/Dead-APC/Cy7, CD4-FITC, CD8-Alexa700, CD94-BV421 and CD69-PeCy7, B220-BV605, CD11b-PerCPCy5.5, and Ly6G-APC (all Biolegend) cells were fixed with $4 \%$ paraformaldehyde (PFA). Sample acquisition was performed on a FACSSymphony-A5 (BD), and data analyzed in FlowJo V10. Cell populations were identified by initially gating on Live/ Dead negative, doublet negative (SSC-H vs SSC-A). Activation positive responses are presented after subtraction of the background responses detected in the LASV GPC peptide pool-stimulated samples.

\section{Virus Neutralization Assay}

The day before this assay, VeroE6 cells were seeded in 96-well plates. Serum samples were heat-inactivated for $30 \mathrm{~min}$ at $56^{\circ} \mathrm{C}$, and 2-fold serial dilutions were prepared in DMEM with 2\% FBS. Next, 100 TCID $_{50}$ of SARS-CoV-2 were added and the mixture was incubated for 1 hour at $37^{\circ} \mathrm{C}$ and $5 \% \mathrm{CO}_{2}$. Finally, media was removed from cells and the mixture was added to VeroE6 cells and incubated at $37^{\circ} \mathrm{C}$ and $5 \% \mathrm{CO}_{2}$ for 6 days. CPE was documented, and the virus neutralization titer was expressed as the reciprocal value of the highest dilution of the serum which inhibited virus replication (no CPE) (43).

\section{Histology and Immunohistochemistry}

Tissues were fixed in $10 \%$ neutral buffered formalin with two changes, for a minimum of 7 days. Tissues were placed in cassettes and processed with a Sakura VIP-6 Tissue Tek, on a 12-hour automated schedule, using a graded series of ethanol, xylene, and ParaPlast Extra. Embedded tissues are sectioned at $5 \mathrm{um}$ and dried overnight at 42 degrees $\mathrm{C}$ prior to staining. Specific anti-CoV immunoreactivity was detected using Sino Biological Inc. SARS-CoV/SARS-CoV-2 N antibody (Sino Biological cat\#40143-MM05) at a 1:1000 dilution. The secondary antibody was the Vector Laboratories ImPress VR anti-mouse IgG polymer (cat\# MP-7422). The tissues were then processed for immunohistochemistry using the Discovery Ultra automated stainer (Ventana Medical Systems) with a ChromoMap DAB kit (Roche Tissue Diagnostics cat\#760-159). All tissue slides were evaluated by a board-certified veterinary pathologist, a representative low (20x) and high (200x) magnification photomicrograph of lung from each group was selected. Lung sections were analyzed for evidence of interstitial pneumonia and assigned the following scores: 0 normal, 1 minimal, 2 mild, 3 moderate, 4 severe.

\section{Statistical Analyses}

All statistical analysis was performed in Prism 8 (GraphPad). The serology, cellular response, RNA levels, titers and growth kinetics were examined using two-way ANOVA with Tukey's multiple comparisons to evaluate statistical significance at all timepoints. Two-tailed Mann-Whitney or Wilcoxon tests were conducted to compare differences between groups for all other data. Statistically significant differences are indicated as $\mathrm{p}<0.0001$ $\left(^{* * *}\right), \mathrm{p}<0.001\left(^{* * *}\right), \mathrm{p}<0.01\left(^{* *}\right)$ and $\left.\mathrm{p}<0.05^{*}\right)$.

\section{RESULTS}

\section{Vaccine Construction and Characterization}

The VSV full-length plasmid encoding the EBOV-Kikwit GP, the primary antigen for the approved EBOV vaccine, was used as the parental vector to construct the COVID-19 vaccines. First, we generated a bivalent VSV construct co-expressing the EBOV GP and SARS-CoV-2 S (VSV-SARS2-EBOV) by adding the fulllength codon-optimized SARS-CoV-2 S upstream of the EBOV GP into the existing VSV vector (Figure S1A). Second, we generated a monovalent VSV construct by replacing the EBOV GP with the SARS-CoV-2 S which contains a cytoplasmic tail deletion previously described $(28,45)$. Both constructs were recovered from plasmid following previously established protocols (42). Expression of both antigens, SARS-CoV-2 S and EBOV GP, was confirmed by Western blot analysis of the VSV particles in cell supernatant (Figure S1B). Next, we performed viral growth kinetics and found that VSV-SARS2EBOV replicated with similar kinetics and had comparable endpoint titers as the parental VSV-EBOV (Figure S1C). In contrast, VSV-SARS2 showed an attenuated growth curve, and the endpoint titer was significantly lower compared to the VSVSARS2-EBOV, potentially impacting vaccine production.

\section{VSV-Based Vaccines Elicit Antigen- Specific Humoral Responses}

Groups of Syrian golden hamsters (Table S1) were vaccinated with $1 \times 10^{5}$ plaque forming units (PFU) either IM or IN with VSV-EBOV (46), VSV-SARS2, or VSV-SARS2-EBOV. Blood samples were collected at $3,10,21$, and 38 days post vaccination (DPV). The humoral immune response to vaccination was examined by enzyme-linked immunosorbent assay (ELISA) using recombinant full-length $S$, recombinantly expressed $S$ receptor binding domain (RBD), and recombinantly expressed EBOV GP. S-specific IgG antibodies were detected 10 DPV in the sera of both the IM- and IN-vaccinated groups for VSV-SARS2 and VSV-SARS2-EBOV (Figures 1A, B) with antibody titers significantly higher in the VSV-SARS-EBOV IM group at 21 and $38 \mathrm{DPV}$ (Figure 1A). Hamsters in the control groups (VSV-EBOV-vaccinated) had no detectable $\mathrm{S}$-specific or S RBD-specific IgG (Figures 1A-D). Similar to 
A

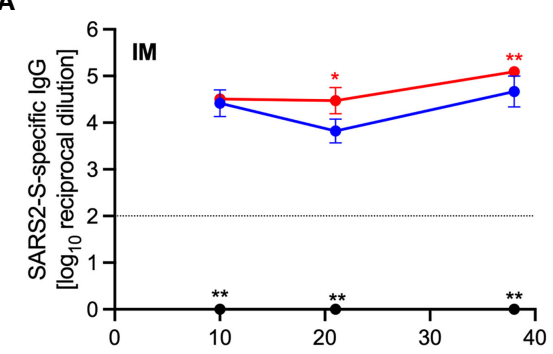

C

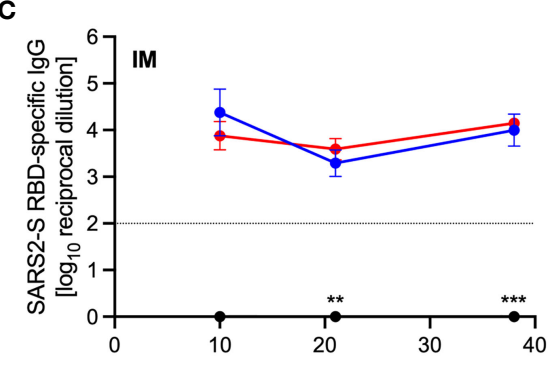

E

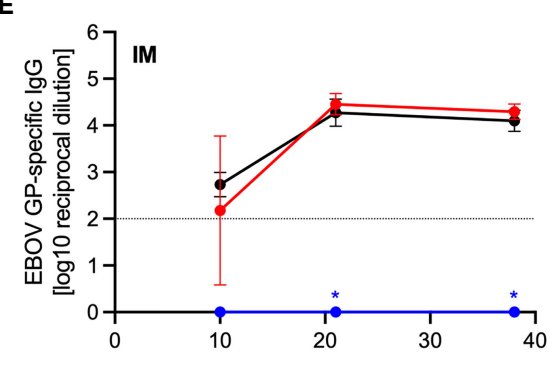

G

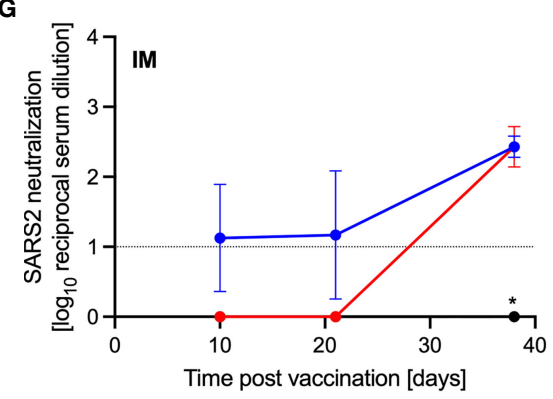

B

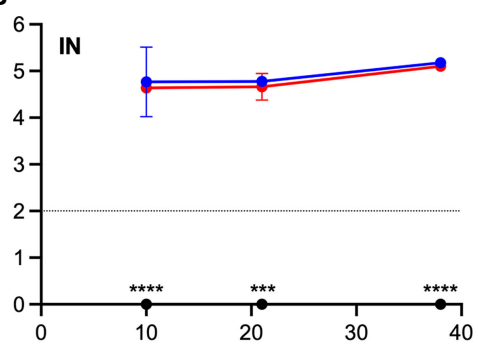

D

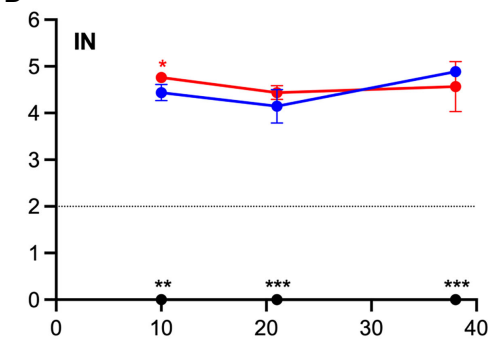

$\mathbf{F}$

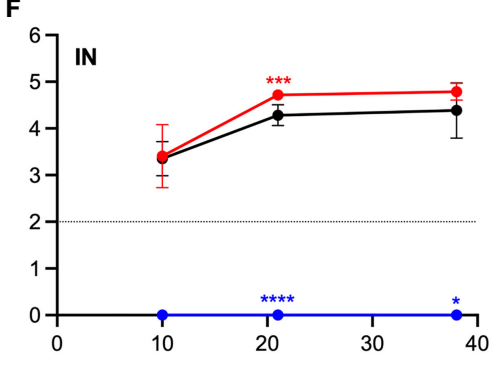

H

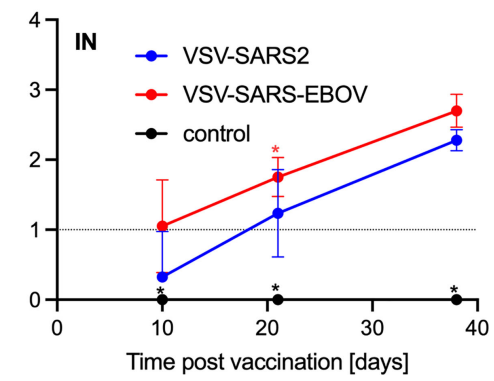

FIGURE 1 | Immunogenicity humoral immune response. Serum samples were collected at multiple time points after vaccination to determine the progression of the antigen-specific antibody response by ELISA. (A, B) SARS-CoV-2 S-specific IgG. (C, D) SARS-CoV-2 S receptor binding domain (RBD)-specific IgG. (E, F) Ebola virus glycoprotein (EBOV GP)-specific IgG. (G, H) SARS-CoV- 2 neutralization. Geometric mean and geometric SD are depicted. Statistical significance as determined by two-way ANOVA with Tukey's multiple comparison is indicated as $p<0.0001\left(^{(\star \star \star}\right), p<0.001\left(^{\star \star \star}\right)$, $p<0.01\left(^{(\star}\right)$, and $p<0.05\left(^{(*)}\right.$.

the S-specific IgG response, all animals vaccinated with VSVSARS2 and VSV-SARS2-EBOV developed measurable antibody titers to the $\mathrm{S} R \mathrm{RDD}$, independent of vaccination route (Figures 1C, D). RBD-specific antibody titers were significantly increased in the VSV-SARS2-EBOV IN-vaccinated animals at 10 DPV only (Figure 1D). Significantly higher antibody titers for EBOV GP were not detected between VSVEBOV and VSV-SARS2-EBOV except for $21 \mathrm{DPV}$ in the IN group only (Figures 1E, F). Antibody functionality was assessed by SARS-CoV-2 neutralization and resulted in no significant difference between the IM-vaccinated groups (Figures 1G, H). Only VSV-SARS2-EBOV IN-vaccinated animals had a significantly higher neutralization titer compared to VSVSARS2 at 21 DPV (Figure 1H). Overall, VSV-SARS2-EBOV elicited a more robust and durable antigen-specific humoral response in hamsters particularly after IN administration. 


\section{VSV-Based Vaccines Induce Limited Cellular Response}

Given the potential role of cellular immunity to contribute to immune protection as seen with SARS-CoV-1 and Middle East respiratory syndrome (43), we sought to use flow cytometry to characterize the cellular populations involved (47-49). Cellular immunology is a particular challenge in the hamster model due to the limited number of reagents available. A panel of mouseand rat-specific flow cytometry antibodies was screened for cross-reactivity to characterize multiple cellular populations (Table S2) and a representative gating strategy is shown (Figure S2). After we identified 7 antibodies that reacted in our initial tests, samples collected on 3, 10, and 38 DPV were used to monitor the change in cellular phenotypes over time. Single cell suspensions were created for the lungs, spleen, and PBMCs and labeled for CD4, CD8, and CD69 to characterize activated and potentially resident memory $\mathrm{T}$ cell populations, CD94 to identify natural killer (NK) cells, as well as B220 to stain for $\mathrm{B}$ cells. We detected a greater percentage of activated $\mathrm{CD} 4^{+} \mathrm{T}$ cells in IM-vaccinated hamsters $3 \mathrm{DPV}$, however, overall CD4 ${ }^{+} \mathrm{T}$ cell responses peaked in the VSV-SARS2-EBOV IN group 10
DPV (Figures 2A, B). There was more overall $\mathrm{CD}^{+} \mathrm{T}$ cell stimulation on 3 and $10 \mathrm{DPV}$ in the IN groups, but significantly more activated lung $\mathrm{CD}^{+} \mathrm{T}$ cells were produced in the same time frame for the IM-vaccinated animals (Figures 2C, D). IMvaccinated animals produced more NK cells on 3 and 10 DPV with minimal effect on B cells (Figures 2E, F). Overall, IM vaccination appeared to elicit a rapid $\mathrm{CD} 4^{+} \mathrm{T}$ cell and $\mathrm{NK}$ cell response, while IN-vaccination resulted in a rapid $\mathrm{CD}^{+} \mathrm{T}$ cell response in the lungs.

We examined the same cellular populations in the spleen and in PBMCs of the vaccinated animals. Peak levels of $\mathrm{CD}^{+} \mathrm{T}$ cells were measured $10 \mathrm{DPV}$ in the spleen after vaccination by both routes, however, IN vaccination induced more $\mathrm{CD} 4^{+} \mathrm{T}$ cells 38 DPV (Figure 3A). In contrast, IM vaccination induced more $\mathrm{CD}^{+} \mathrm{T}$ cells on 3 and $10 \mathrm{DPV}$ (Figure 3C). No to limited activated $\mathrm{CD}^{+}$or $\mathrm{CD}^{+} \mathrm{T}$ cell responses were detected (Figures 3B, D). While IN vaccination resulted in greater numbers of NK cells on 3 and 10 DPV and in more B cells 3 DPV, IM vaccination induced higher numbers of NK cells on 38 DPV and B cells 10 and 38 DPV (Figures 3E, F). PBMCs of INvaccinated animals demonstrated higher levels of $\mathrm{CD}^{+} \mathrm{T}$ cells

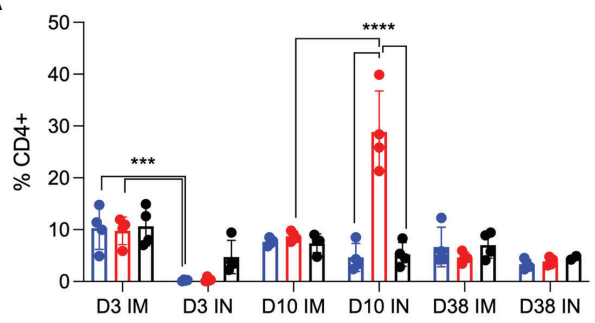

C

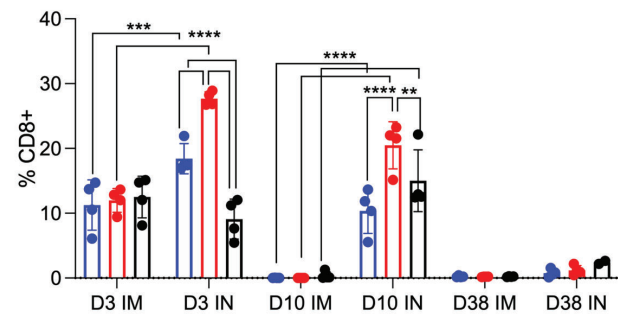

E

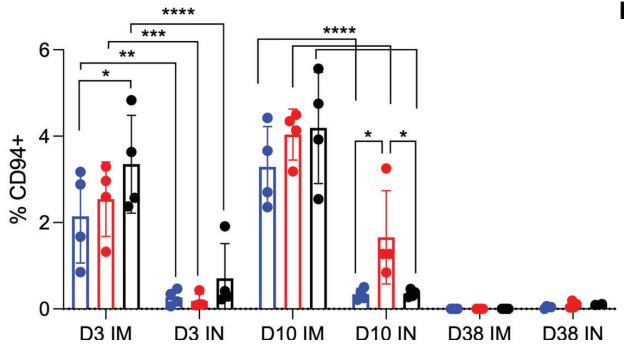

B

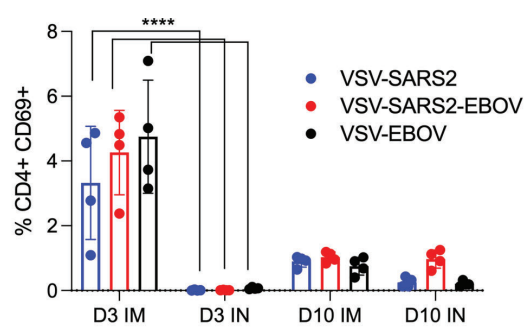

D
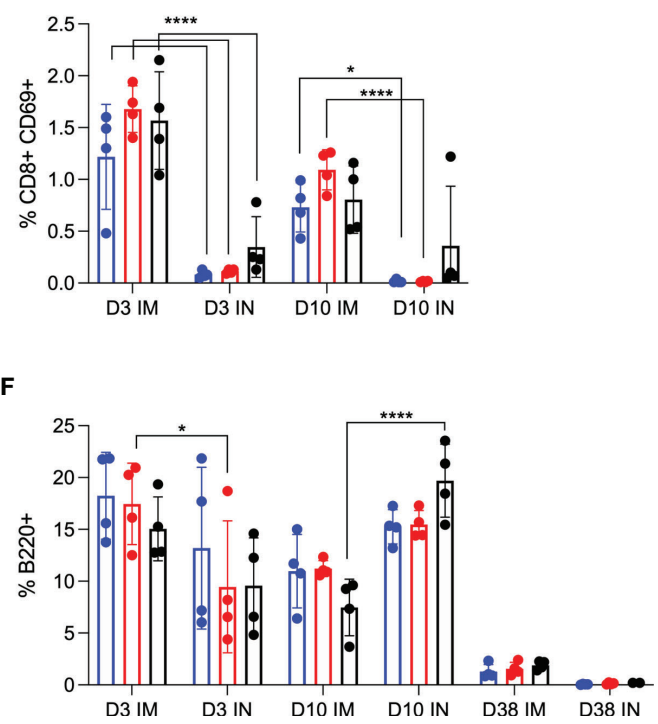

FIGURE 2 | Immunogenicity cellular immune response in the lungs. Single cell lung suspensions were stained for FACS analysis. (A, B) CD4 ${ }^{+}$T cells and (C, D) CD8 ${ }^{+}$ T cells were identified and stained for expression of early activation marker CD69. (E) NK cells were identified and stained for expression of CD94. (F) B cells were identified and stained for expression of B220. Mean and SD are depicted. Statistical significance determined by two-way ANOVA with Tukey's multiple comparison is indicated as $\left.p<0.0001^{\left({ }^{* \star}\right)}, p<0.001^{(\star \star}\right), p<0.01\left(^{\star *}\right)$, and $p<0.05^{(*)}$. 


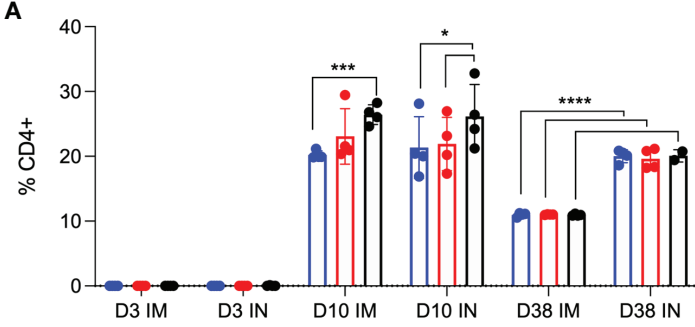

C

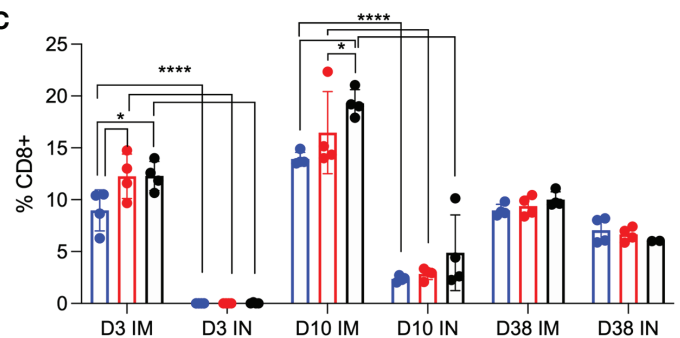

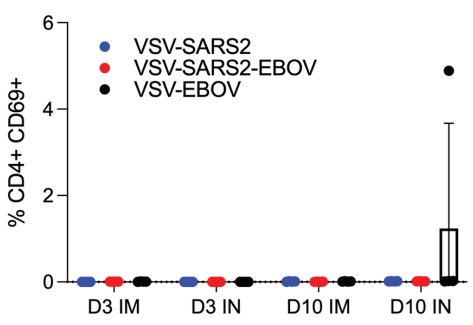

D

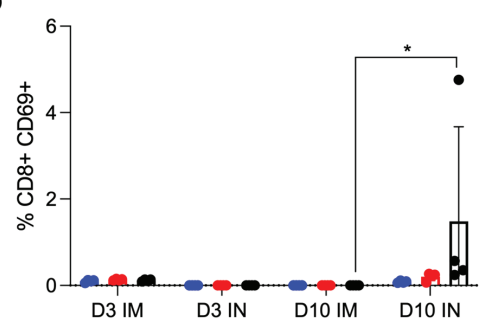

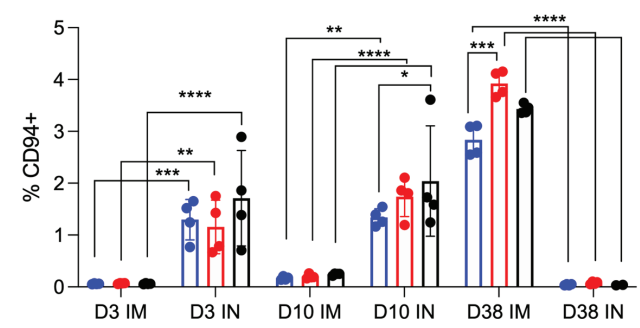

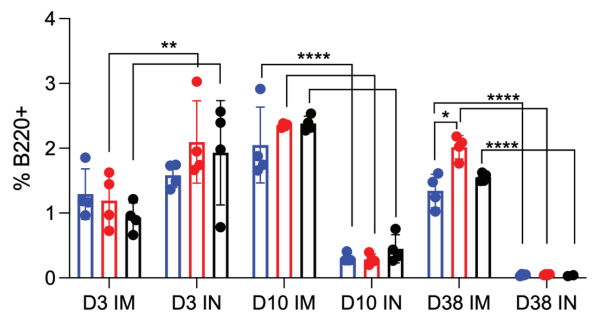

FIGURE 3 | Immunogenicity cellular immune response in the spleen. Single cell splenocyte suspensions were stained for FACS analysis. (A, B) CD4 ${ }^{+}$T cells and (C, D) CD8 ${ }^{+} T$ cells were identified and stained for expression of early activation marker CD69. (E) NK cells were identified and stained for expression of CD94. (F) B cells were identified and stained for expression of B220. Mean and SD are depicted. Statistical significance determined by two-way ANOVA with Tukey's multiple comparison is indicated as $\left.\left.p<0.0001^{(\star \star \star}\right), p<0.001^{(* \star}\right), p<0.01_{(* \star}^{(*)}$, and $p<0.05\left(^{\star}\right)$.

on $38 \mathrm{DPV}$, while IM vaccination induced significantly more activated $\mathrm{CD} 8^{+} \mathrm{T}$ cells on $10 \mathrm{DPV}$ and $\mathrm{CD} 4^{+} \mathrm{T}$ cells and NK cells on 38 DPV (Figures S3A-F).

\section{VSV-Based Vaccines Protect Hamsters From COVID-19 Within 10 Days}

For initial efficacy study in hamsters, we vaccinated groups of 8 animals (4 female and 4 male) with $1 \times 10^{5}$ PFU either IM or IN with VSV-EBOV (46), VSV-SARS2, or VSV-SARS2-EBOV. The animals were challenged with $1 \times 10^{5} \mathrm{TCID}_{50}$ of the SARS-CoV-2 WA1 isolate $28 \mathrm{DPV}$ (day 0) and euthanized 4 DPC for sample collection. Oral swab samples at the time of necropsy revealed no significant differences in viral shedding as determined by RT-qPCR (Figure 4A). In contrast, lungs from all vaccinated hamsters presented without lesions (Figures S4A, B, D, E) and a significant decrease in lung virus loads determined by RT-qPCR (Figure 4B) and titration (Figure 4C). All control animals presented with gross lung lesions (Figures S4C, F) and high lung virus loads (Figures 4B, C). When we investigated the antibody response $4 \mathrm{DPC}$, we found higher S-specific IgG titers after both routes of vaccination, however, only titers after IN vaccination were statistically significant
(Figure 4D). Neutralization against the SARS-CoV-2 WA1 isolate revealed significantly higher titers for all vaccinated groups compared to control hamsters (Figure 4E). In addition, the VSVSARS2-EBOV vaccine resulted in significantly higher titers after IN administration compared to VSV-SARS2 (Figure 4E).

Next, we explored the fast-acting potential of these vaccines by shortening the time between vaccination and challenge to 10 days. Because we did not observe a difference between male and female hamsters in the previous experiment, all following experiments were conducted using female hamsters only. Groups of 6 hamsters were vaccinated with $1 \times 10^{5}$ PFU with VSV-EBOV (46), VSVSARS2, or VSV-SARS2-EBOV either IM or IN. The animals were challenged with $1 \times 10^{5} \mathrm{TCID}_{50}$ of the SARS-CoV-2 WA1 isolate 10 DPV (day 0) and euthanized 4 DPC for sample collection. Oral swabs demonstrated a significant decrease in viral RNA indicating reduced shedding in vaccinated animals (Figure 5A). Gross lung pathology revealed lesions in the control animals (Figures S4I, L) and, to a lesser extent, in the VSV-SARS2 IM group (Figure S4G). Hamsters vaccinated with VSV-SARS-EBOV presented without lung lesions (Figures $\mathbf{S 4 H}, \mathbf{K}$ ) as did the VSV-SARS2 INvaccinated group (Figure S4J). Viral loads in the lungs revealed 
A

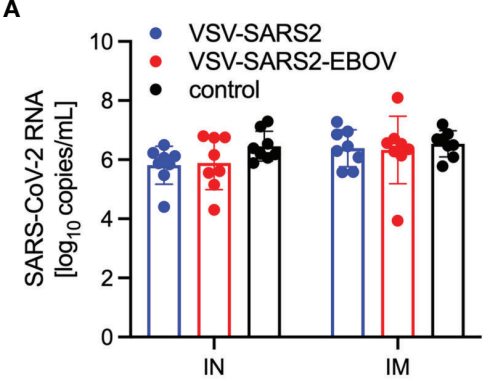

D

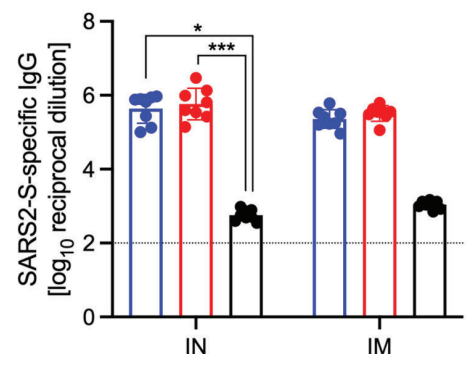

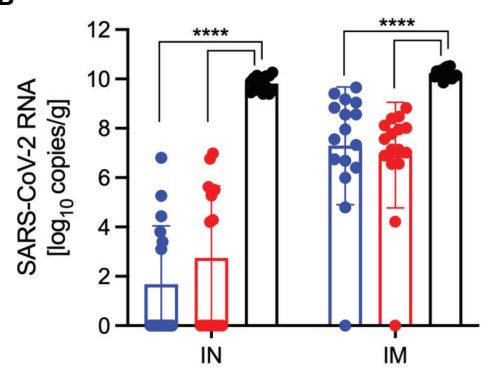

E

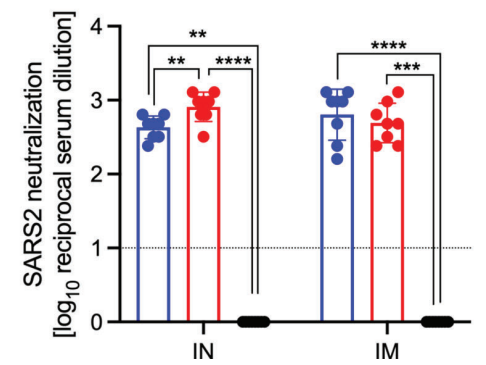

C

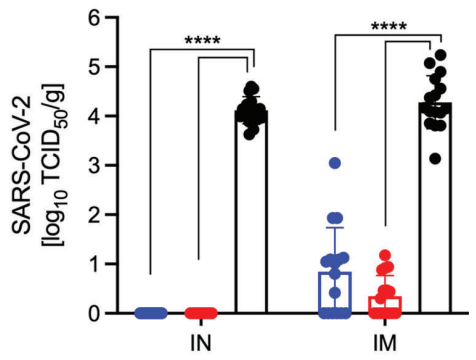

FIGURE 4 | Virus loads and antibody levels in hamsters challenged 28 days post-vaccination. Hamsters were vaccinated with a single dose intramuscularly (IM) or intranasally (IN) 28 days prior to challenge with SARS-CoV-2 WA1. At 4 days after challenge oral swab, lung and serum samples were collected. Levels of SARSCoV-2 RNA in (A) oral swabs and (B) lung samples. (C) Virus titer in hamster lungs. (D) SARS-CoV-2 S-specific IgG and (E) neutralizing titers against SARS-CoV-2 WA1 are shown. Geometric mean and geometric SD are depicted. Statistical significance as determined by two-way ANOVA with Tukey's multiple comparison is indicated as $\left.\left.p<0.0001^{(\star \star \star}\right), p<0.001^{(\star \star}\right), p<0.01\left(^{(\star}\right)$, and $p<0.05\left(^{(*)}\right.$.

significant differences between vaccinated and control animals by RT-qPCR and virus titration (Figures 5B, C). Histopathologic analysis of lung samples collected 4 DPC demonstrated evidence of interstitial pneumonia in all control animals (Figures 6I, K) and was quantified by the application of a pathology score (Figure 5D). While interstitial pneumonia was significantly reduced in the animals vaccinated IN with both vectors or IM with VSV-SARS2EBOV (Figures 5D, 6A, C, G), lung sections of animals in the VSVSARS2 IM group showed evidence of broncho-interstitial pneumonia consistent with coronaviral pulmonary disease (Figure 6E). Immunohistochemistry (IHC) revealed the presence of SARS-CoV-2 N in the lungs of control animals only (Figures 6J, L) indicating control of virus replication in all vaccine groups (Figures 6B, D, F, H). Analysis of S-specific IgG in the serum of hamsters 4 DPC demonstrated significantly higher S-specific IgG titers after both routes of vaccination (Figure 5E). Neutralization against the SARS-CoV-2 WA1 isolate revealed significantly higher titers for VSV-SARS2 IN, VSV-SARS2-EBOV IN and VSV-SARS2EBOV IM vaccine groups compared to the control group (Figure 5F). In addition, the VSV-SARS2-EBOV vaccine resulted in significantly higher titers after IM administration compared to VSV-SARS2 (Figure 5E).

\section{IN Vaccination with VSV-Based Vaccines Protects Hamsters Against Infection with VOC}

SARS-CoV-2 VOC are in the focus of efficacy testing for approved vaccines. Therefore, we investigated the protective potential of our VSV-based vaccines against two VOC: B.1.1.7 and B.1.351. Groups of hamsters were vaccinated with $1 \times 10^{5}$ PFU VSV-EBOV (46), VSV-SARS2, or VSV-SARS2-EBOV (Table S2). VSV-SARS2 IM vaccination was not protective as described above, thus we omitted this group. The hamsters were challenged with $1 \times 10^{5} \mathrm{TCID}_{50}$ of the SARS-CoV-2 B.1.1.7 or B.1.351 10 DPV (day 0) and euthanized 4 DPC for sample collection. Oral swabs taken from vaccinated hamsters showed reduced levels of viral RNA compared to the control groups, however, the differences were not significant for either VOC (Figure 7A). Gross pathology of the lungs at the time of necropsy 4 DPC revealed lesions in the control groups for both VOC (Figures S5D, H). Animals in the VSV-SARS2-EBOV IM group presented with limited lung lesions after B.1.1.7 infection (Figure S5B), whereas VSV-SARS2 or VSV-SARS2-EBOV INvaccinated hamsters did not show any lesions grossly (Figures S5A, C). For the challenge with B.1.351 only hamsters INvaccinated with VSV-SARS2 presented with non-lesioned lungs (Figure S5G). Lung virus loads supported the gross pathology observations for B.1.1.7 challenge with lowest viral RNA detected after IN vaccination (Figure 7B). Similarly, only IN vaccination significantly reduced SARS-CoV-2 RNA in the lungs of B.1.351-infecetd hamsters albeit to lower extent when compared to B.1.1.7-infected hamsters (Figure 7B). Virus titration of lung samples confirmed the RNA data demonstrating significantly reduced SARS-CoV-2 levels after B.1.1.7 challenge in all vaccinated animals and after B.1.351 challenge in IN-vaccinated hamsters (Figure 7C). 
A

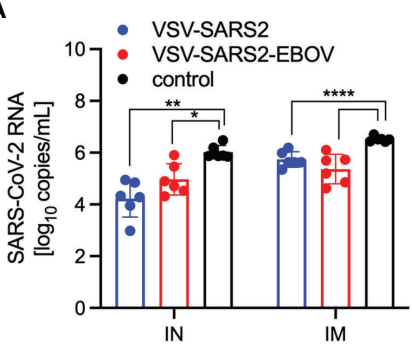

D

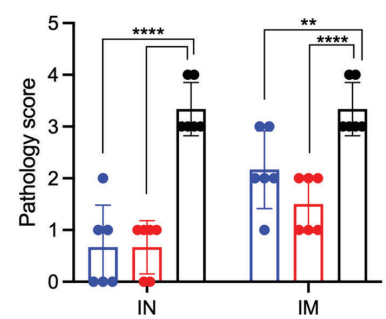

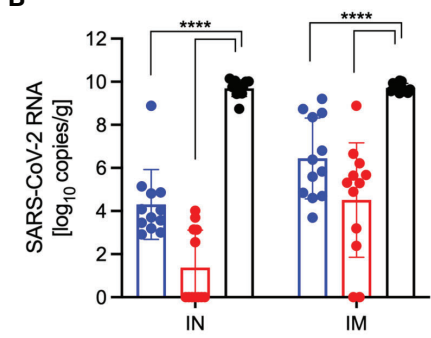

E

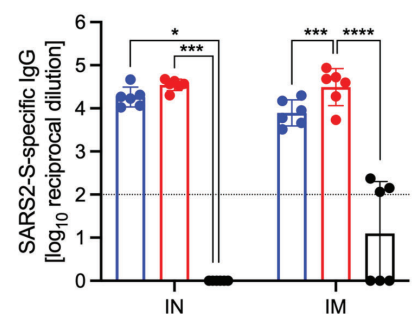

C

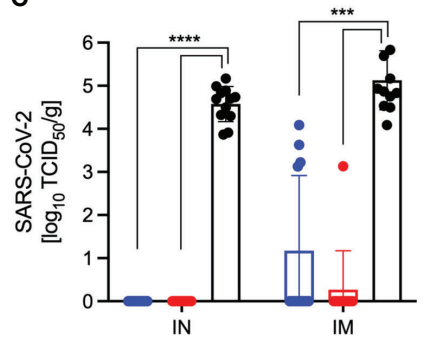

$\mathbf{F}$

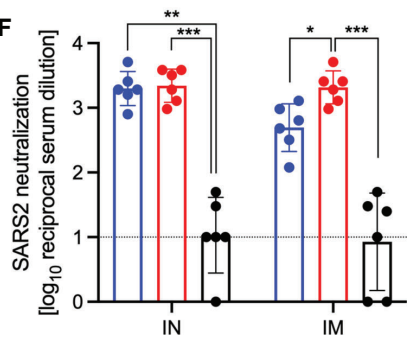

FIGURE 5 | Virus loads and antibody levels in hamsters challenged 10 days post-vaccination. Hamsters were vaccinated with a single dose intramuscularly (IM) or intranasally (IN) 10 days prior to challenge with SARS-CoV-2 WA1. At 4 days after challenge oral swab, lung and serum samples were collected. Levels of SARSCoV-2 RNA in (A) oral swabs and (B) lung samples. (C) Virus titer in hamster lungs. (D) Lung sections were scored for evidence of interstitial pneumonia (1= minimal, $2=$ mild, $3=$ moderate, and 4= severe). (E) SARS-CoV-2 S-specific lgG and (F) neutralizing titers against SARS-CoV-2 WA1 are shown. Geometric mean and geometric SD are depicted. Statistical significance as determined by two-way ANOVA with Tukey's multiple comparison is indicated as $p<0.0001$ ( $\left.{ }^{\star \star \star \star}\right), p<$ $0.001\left(^{\star \star \star}\right), p<0.01\left(^{\star \star}\right)$, and $p<0.05\left(^{\star}\right)$.

Histopathology revealed significant reductions of evidence of broncho-interstitial pneumonia for IN-vaccinated hamsters with increased vaccination efficacy in the B.1.1.7 group (Figure 7D). Representative lung sections for each group indicated that VSVSARS2-EBOV IN vaccination was the most efficacious vaccine against VOC challenge with limited pathological changes and no presence of viral antigen (Figure S6). Antigen-specific IgG responses were examined from $4 \mathrm{DPC}$ and demonstrated significant titers in all vaccinated hamsters compared to the control groups (Figure 7E). While there was no significant difference in vaccinated and B.1.1.7-infecetd hamsters for Sspecific IgG or neutralization activity (Figures 7E, F), vaccination with VSV-SARS2-EBOV IN and B.1.351 challenge resulted in a significantly higher S-specific IgG titer (Figure 7E). Interestingly, this difference could not be confirmed in the neutralization assay. Serum of hamsters vaccinated with VSVSARS2 IN and challenged with B.1.351 had the highest neutralizing titers against B.1.351 (Figure 7F). Because SARSCoV-2 variant B.1.617.2 (Delta) is currently the dominating circulating variant, we determined if serum samples from the day -10 vaccination studies for the VOC shown here had the ability to cross-neutralize this variant. We observed a significant increase in the neutralization titer of VSV-SARS2 IN after both VOC challenges and in the VSV-SARS2-EBOV IN group after B.1.351 challenge compared to the control (Figure 7G). In order to analyze if this response is similar to cross-neutralization observed in hamsters infected with B1.1.7 or B.1.351 after 14 or 28 days, we utilized serum samples from a previous study conducted in the lab (48) and performed neutralization assays. We found that our vaccinated and challenged hamsters presented with an increased neutralizing capacity for B.1.617.2 compared to hamsters infected with VOC for 14 days, particularly infected with B.1.351 (Figure 7H). By day 28, the VOC-infected hamsters have a similar neutralizing response compared to our animals. This data demonstrates that IN vaccination with VSV-based vaccines expressing SARS-CoV-2 $S$ is efficacious against VOC infection within 10 days, and that vaccination induced significantly higher neutralization titers than if an animal was infected and recovered from disease.

\section{DISCUSSION}

The COVID-19 pandemic is not slowing down and surges in cases caused by VOC are ongoing. The most efficient way to stop the pandemic is vaccination. An effective COVID-19 vaccine would ideally induce protective immunity rapidly after only a single dose, thus reducing the pressure on vaccine production and the healthcare system. Given that VSV-based vaccines often require only a single dose to be effective while inducing a rapid and robust immune response, they offer considerable potential to meet this need. Most of the SARS-CoV-2 vaccines that have been authorized for emergency human use utilize adenovirus- or mRNA-based platforms that require a prime and boost vaccination schedule to fully generate protective immunity (18, 19). The prime/boost vaccination strategy requires significant time to achieve full immunity, which intrinsically puts patients at risk. Our goal was to generate a fast-acting single-dose vaccine that could be implemented in an emergency situation for naïve people or as a fast-acting booster vaccination for previously 

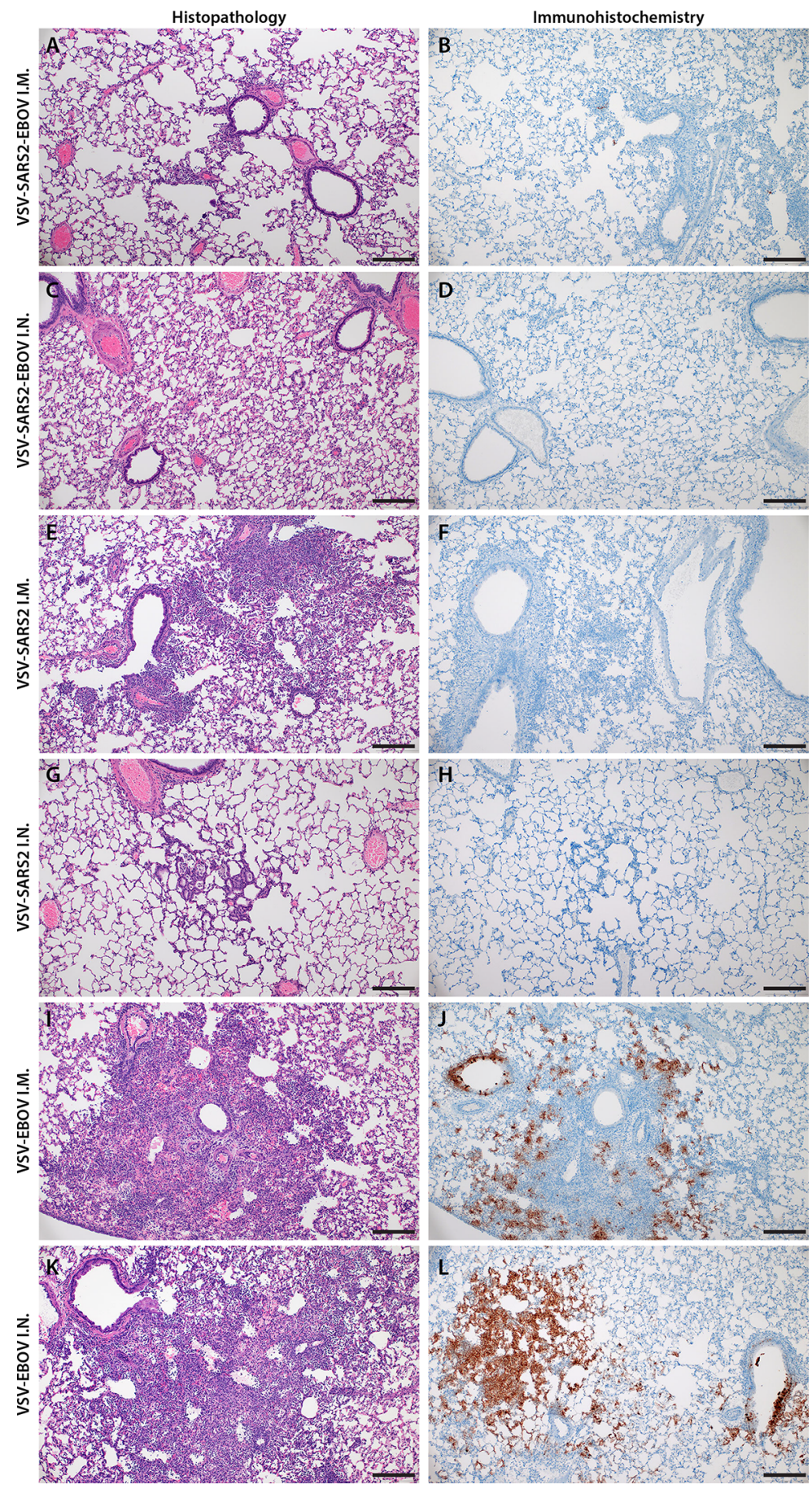

FIGURE 6 | Histopathology and Immunohistochemistry of hamster lungs with challenge 10 DPV. Hamsters were vaccinated 10 days prior to challenge with SARSCoV-2 WA1. At 4 days after challenge lung samples were collected and stained with H\&E or anti-SARS-CoV-2 nucleocapsid (N) antibody for IHC. (A) Rare foci of minimal to mild interstitial pneumonia with mild alveolar spillover. (B) Rare type I pneumocyte immunoreactivity. (C) Lack of notable pulmonary histopathology. (D) No immunoreactivity to SARS-CoV-2 N. (E) Focus of mild to moderate broncho-interstitial pneumonia with perivascular leukocyte cuffing. (F) Limited type I pneumocyte immunoreactivity. (G) Rare foci of minimal to mild interstitial pneumonia with type II pneumocyte hyperplasia. (H) No immunoreactivity to SARS-CoV-2 N. (I) Focus of moderate to severe bronchointerstitial pneumonia with disruption of pulmonary architecture by degenerate and non-degenerate neutrophils, macrophages and cellular debris accompanied with perivascular and pulmonary edema. (J) Abundant immunoreactivity to SARS-CoV-2 N in columnar epithelium of bronchioles, type I pneumocytes and alveolar macrophages. (K) Moderate broncho-interstitial pneumonia with influx of moderate to numerous leukocytes and limited pulmonary edema. (L) Abundant immunoreactivity to SARS-CoV-2 N in bronchiolar epithelium, type I and II pneumocytes and within cellular debris. (200x, bar = 50 $\mu$ M). 
A

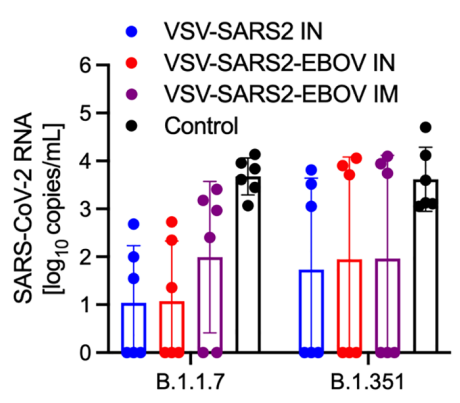

D

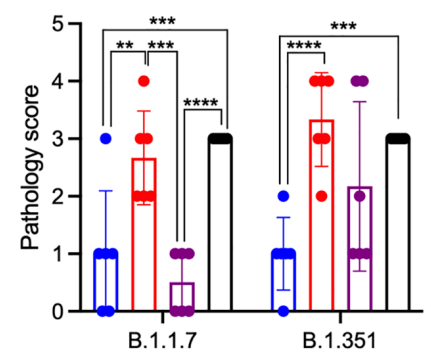

G

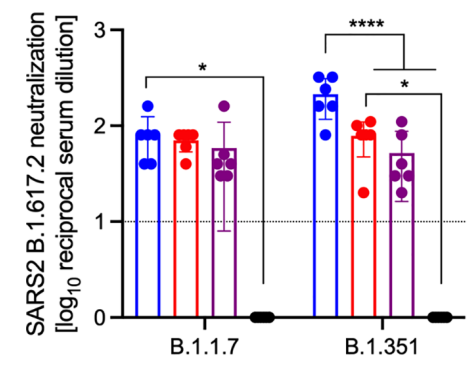

B

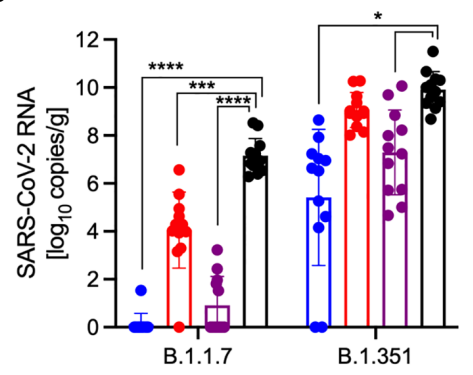

E

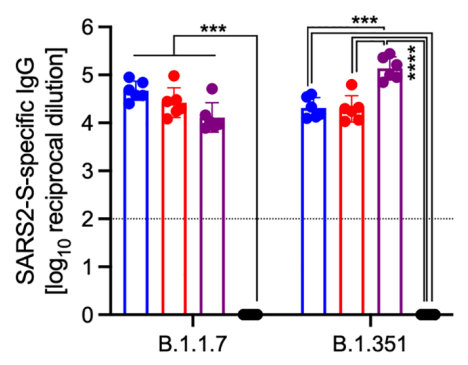

H

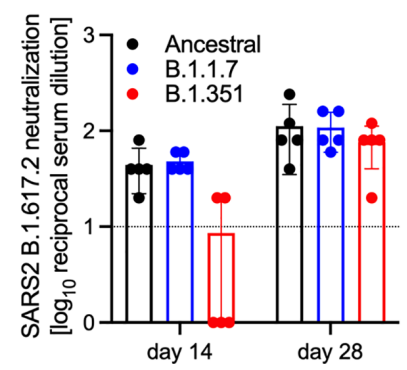

C

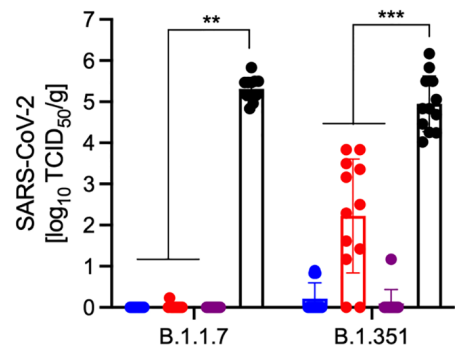

$\mathbf{F}$

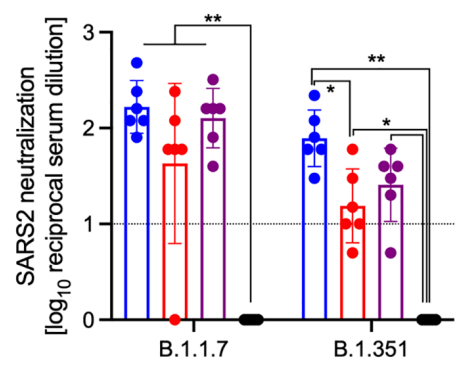

FIGURE 7 | Virus loads and antibody levels in hamsters challenged 10 days post-vaccination with VOC. Hamsters were vaccinated with a single dose intramuscularly (IM) or intranasally (IN) 10 days prior to challenge with SARS-CoV-2 B.1.1.7 or B.1.351. At 4 days after challenge oral swab, lung and serum samples were collected. Levels of SARS-CoV-2 RNA in (A) oral swabs and (B) lung samples. (C) Virus titer in hamster lungs. (D) Lung sections were scored for evidence of interstitial pneumonia (1= minimal, 2= mild, 3= moderate, and 4= severe). (E) SARS-CoV-2 S-specific lgG and (F) neutralizing titers against SARS-CoV-2 WA1 are shown. (G) Neutralizing titers against SARS-CoV-2 B.1.617.2 are shown. (H) Serum from hamsters infected with SARS-CoV-2 WA1 (Ancestral), B.1.1.7 or B.1.351 for 14 or 28 days were tested for neutralization of SARS-CoV-2 B.1.617.2. (A-C, E-H) Geometric mean and geometric SD or (D) mean and SD are depicted. Statistical significance as determined by two-way ANOVA with Tukey's multiple comparison is indicated as $p<0.0001\left(^{\star \star \star *}\right), p<0.001\left(^{\star \star \star}\right), p<0.01\left(^{(\star)}\right)$, and $p<0.05\left(^{*}\right)$.

vaccinated individuals or COVID-19 survivors who have waning immunity.

Despite being a live-attenuated vaccine, the VSV vaccine platform has several attributes that contribute to its safety profile, an important consideration for a newly developed vaccine. First, the VSV-based vaccines lack the VSV glycoprotein which is considered the primary virulence factor (50). Additionally, VSV is sensitive to interferon $\alpha / \beta$ and an intact innate immune system is able to control VSV replication (31). Lastly, the VSV-SARS2-EBOV vector is based on the FDAand EMA-approved EBOV vaccine by Merck and further attenuated by the addition of SARS-CoV-2 S, another safety feature. However, proper toxicity studies for this vector will still be needed for licensure.
The vaccines described here demonstrated strong efficacy regardless of vaccination route when a "classical" 28-day vaccination to challenge model was utilized. When a shorter time to challenge was implemented, the route of vaccination greatly affected the vaccine efficacy with VSV-SARS2 only being effective by the IN route against all three tested viruses. Merck developed a VSV-SARS2 vaccine similar to ours, but recently halted the production because a phase 1 clinical trials demonstrated humoral antigen-specific responses below the levels of COVID-19 survivors following well-tolerated IM administration (51). The report mentions that alternative routes of vaccination including IN are still being investigated, which reflect our data showing increased vaccine efficacy via IN administration. The poor performance of the vaccine may be due 
to the fact that VSV-SARS2 can only infect ACE2 expressingcells at the site of vaccination. IN vaccination may be more successful due to the abundant expression of ACE2 in the nasal mucosa compared to fewer in muscle tissue (52). In contrast, our bivalent VSV-SARS2-EBOV vaccine was effective both after IN and IM administration. The bivalent vaccine expresses a fulllength S in contrast to VSV-ASRS2 which harbors a 19 amino acid deletion in the cytoplasmic tails of $\mathrm{S}$. The deletion is very likely not associated with the difference we observe as for $\mathrm{S}$ the immunogenic domains including $\mathrm{RBD}$ are located on the extracellular part of the protein. Our findings using the bivalent vaccine highlight the potential for the use of two glycoproteins with different cellular affinities to promote early replication in different anatomical areas. Interestingly, this is in contrast to the data we have generated in NHPs, where IM VSV-SARS2-EBOV vaccination was more efficacious than IN when 10 days between vaccination and challenge were tested (36).

The Syrian golden hamster model is a highly susceptible model for SARS-CoV-2, with an $\operatorname{ID}_{50}$ of 5 TCID $_{50}$ (38). Viral RNA and infectious viral titers are high in the respiratory tract of infected hamsters, but do not translate to severe clinical disease manifestations with hamsters displaying minimal weight loss and no to minor outward signs of disease. However, when lung samples are analyzed, histopathology shows evidence of broncho-interstitial pneumonia present in the challenged animals 4 DPC $(38,53)$. Lung pathology resolved when animals were necropsied 14 and 28 DPC indicating that SARSCoV-2 infection is a self-limiting disease in this model system (53). The inhibition of severe lung lesions and signs of interstitial pneumonia early during infection is being used as an indicator of vaccine or antiviral therapy efficacy in the hamster model (38). Histopathologic analysis of lung samples from hamsters vaccinated with either VSV-SARS2 or VSV-SARS2-EBOV demonstrated that regardless of route of immunization, VSVSARS2-EBOV showed minimal pathological changes. Additionally, no viral antigen was present as shown by IHC. Similarly, lungs of hamsters receiving VSV-SARS2 IN presented with minimal pathological features and no viral antigen was detected. In contrast, lungs of hamsters IM-vaccinated with VSV-SARS2 presented with evidence of interstitial pneumonia and viral antigen was detected within foci of pathology. This led us to conclude that VSV-SARS2-EBOV was a superior vaccine candidate particularly when the vaccine was administered only 10 days prior to challenge, however, protective efficacies are likely comparable when administered 28 days prior to challenge.

With the continued emergence of new SARS-CoV-2 VOC harboring mutations that either increase transmissibility or allow for increased evasion from the previously established humoral response, new challenges arise. Existing vaccination strategies and routes of administration must be analyzed to determine the retention of vaccine efficacy against multiple VOC. The two primarily distributed vaccines by Pfizer (BNT162b2) and Moderna (mRNA-1273) have been assessed for sustained efficacy against VOC. A recent report utilizing human serum samples and a pseudovirus neutralization assay determined that vaccination with either mRNA vaccine resulted in moderate decreases in cross-neutralization activity against B.1.1.7. When the cross-reactivity against B.1.351 was assessed the neutralization potential was decreased up to 42.2- (Pfizer) and 27.7- (Moderna) fold, respectively (54). In a meta-analysis review both vaccines had various results against B.1.1.7 with a range of 2.6-fold decrease to 3.8-fold increase in live-virus neutralization for the Pfizer vaccine, and a 1.77-fold decrease to 1.6-fold increase for the Moderna vaccine. In contrast, the cross-neutralization of B.1.351 was significantly impacted with 22.8- (Pfizer) and 12.4(Moderna) fold decreases in live-virus neutralization assays (55). The adenovirus-based vaccine from AstraZeneca ChAdOx1 nCOV/AZD1222 followed a similar trend with 70.4-74\% percent efficacy against B.1.1.7, but only $10.4-22 \%$ efficacy against B.1.351 (56-58). Our studies highlight the importance of such experiments. The primary VSV-SARS2-EBOV vector is highly efficacious against the original virus (WA1) within 10 days. When vaccinated hamsters were challenged with the heterologous B.1.1.7 variant the vaccine remained similarly efficacious. However, when the vaccinated hamsters were challenged with the B.1.351 variant, VSV-SARS2-EBOV efficacy was decreased resulting in hamsters' histopathologic lesions consistent with COVID-19. Interestingly, IN vaccination with VSV-SARS2 was more efficacious against B.1.351 challenge in hamsters within 10 days. However, virus shedding was not reduced after vaccination and challenge similar to other vaccines $(59,60)$. Future studies will decipher if a longer time between vaccination and challenge results in increased protective immunity after vaccination with both vaccines against challenge with SARS-CoV-2 VOC.

VSV-based vaccines primarily elicit humoral immune responses conferring protection from disease (25-27). However, we wanted to determine if cellular responses differ based on route of vaccination. The comparison of the immunological characteristics of the different samples assessed in this study is depicted in Figure S7. The cellular response showed an early $\mathrm{CD} 4{ }^{+} \mathrm{T}$ cell and NK cell response in the lungs of IM-vaccinated hamsters and an early $\mathrm{CD} 8^{+} \mathrm{T}$ cell response in the lungs of IN-vaccinated hamsters. In the spleen, IM vaccination promoted an early $\mathrm{CD} 8^{+} \mathrm{T}$ cell response and a late $\mathrm{NK}$ and $\mathrm{B}$ cell response, while IN vaccination induced an early NK and $\mathrm{B}$ cell response and a late $\mathrm{CD} 4^{+} \mathrm{T}$ cell response. There was little measured activation of $\mathrm{T}$ cells in the spleen of vaccinated hamsters and little involvement of neutrophils in either the lung or the spleen. IM vaccination induced a late circulating NK cell response. IN vaccination induced an early circulating $\mathrm{CD}^{+} \mathrm{T}$ cell response and a late circulating $\mathrm{CD} 4^{+} \mathrm{T}$ cell and $\mathrm{B}$ cell response accompanied by a more robust antibody response. Thus, it appeared that the primary component of the cellular immune response to vaccination with VSV-SARS2 and VSVSARS2-EBOV is centralized around T cells and NK cells. While the activation marker measured did not show a robust response in the spleen of vaccinated animals, the potential for other effector functions such as stimulation of various cytokines could be present and is an area of interest for future research. An additional caveat, the activation marker CD69 is also a 
marker of tissue-resident memory T cells $(39,61,62)$, but at this point there are no additional markers to investigate this observation in detail.

The levels of S-specific IgG measured during the immunogenicity study indicated that both vaccines elicit significantly higher antigen-specific titers compared to control vaccinated animals regardless of vaccination route. This trend also translated to the RBD-specific titers except for $10 \mathrm{DPV}$ in the IM groups. These data indicate that IN vaccination induced a faster and more specific humoral response to potentially neutralizing epitopes. When the functionality of the humoral response was assessed at these time points IN vaccination induced significantly higher neutralization titers 10 and 28 DPV. The humoral response for SARS-CoV-2-S-specific IgG 10 DPV was only significant for VSV-SARS2 when administered IN, while VSV-SARS2-EBOV had significantly higher antigen specific titers compared to control-vaccinated animals regardless of vaccination route. This trend also translated to higher neutralization titers, indicating that not only does VSV-SARS2EBOV generate higher amounts of antigen-specific antibodies, but also more functional antibodies. The differences in humoral responses were abrogated when hamsters were challenged 28 DPV. The overall humoral response post-challenge compared to vaccination alone elicited a 5-10-fold increase in the response, which may be attributed to the boosting effect of the animals' immune system seeing the vaccine antigen for a second time. The overall antigen-specific IgG titers and neutralizing antibody titers 10 and 28 DPV were similar to those reported for ChAdOx1nCOV/AZD1222 when administered as a single dose 28 days prior to challenge in the hamster model. With the limited immunological tools available for the hamster model the route of vaccination dictates the skew of the cellular response for either vaccine. The overall humoral response is stronger for the VSVSARS2-EBOV vaccine, which is reflective of the pathologic findings, particularly the IM vaccination. While not statistically significant, interstitial pneumonia scores never reached the same severity with VSV-SARS2-EBOV as were observed with VSVSARS2. Traditionally VSV vaccination has been more reliant on a strong humoral response to mediate protection, which leads us to conclude that the differences in SARS-CoV-2-S-specific IgG and neutralization titers are of more importance than the difference in the cellular changes due to the route of vaccination.

While our studies provided insightful findings into the protective efficacy of VSV-based vaccines against COVID-19 in the hamster model, there are limitations. First, the hamster model for SARS-CoV-2 does not recapitulate lethal disease manifestations but rather a self-limiting disease regardless of the variants utilized for challenge. While this is representative of most cases of human COVID-19, evaluation of protective efficacy does depend on readouts other than survival. In addition, using this model we cannot determine if the vaccines tested here suppress the extrapulmonary pathologies associated with human COVID-19 such as the brain, liver, kidney, and heart. The route of administration of both the mucosal vaccine and challenge virus in the hamster is limited to IN in our studies due to animal size and experimental setup. In contrast, NHPs can be vaccinated and challenged via multiple experimental routes including conjunctival and intratracheal which was done in our previously mentioned NHP study and might have contributed to the lack of IN protection we observed in NHPs within 10 days (36). The differences in route of vaccination and subsequent immune response have not been fully elucidated in these two animal models. Similarly, immune responses after challenge virus installation by the same route in different animal models requires further investigation. The biggest limitation is the fact that there are only limited immunological reagents for hamsters available making an in-depth analysis of cellular immunity impossible. Despite these limitations, our data indicates strong efficacy to inhibit virus-induced pathology in the lungs, however, our vaccines do not significantly inhibit viral shedding when the vaccines were administered 28 and 10 days prior to challenge by either route. Ideally, vaccination should result in a reduction in viral shedding indicated by decreased virus presence in the oral swabs to not only limit severe disease manifestation, but also to reduce the transmission potential. Therefore, our continued development of next-generation VSV-based vaccines will also focus on the reduction of virus shedding.

Taken together, we generated two effective, single-dose vaccines against COVID-19 efficacious within 10 days in a Syrian golden hamster vaccine-challenge model. VSV-SARS2EBOV is effective 28 and $10 \mathrm{DPV}$, regardless of route of vaccination. Our results suggest that $\mathrm{IN}$ is the optimal route of vaccination in the hamster model for VSV-based vaccines as well as other vaccines (23). Future studies will address the impact of preexisting immunity to SARS-CoV-2 S or EBOV GP in our vaccine, however, we do not anticipate a major effect as both antigens are able to drive the replication of the vaccine virus. However, preexisting immunity may lead to an adjustment of the administered vaccine dose which remains to be investigated (46, $63)$. Furthermore, we will investigate the addition of another SARS-CoV-2 antigen into the vaccine to promote a stronger $\mathrm{T}$ cell response, as these responses are typically longer lasting. At this time, the VSV vaccines presented here have a high potential as a boosting option after the already approved vaccines due to their fast-acting potential and the elicitation of primarily a humoral response in contrast to the predominantly $\mathrm{T}$ celldriven immune response after adenovirus- and mRNA-based vaccination (18).

\section{DATA AVAILABILITY STATEMENT}

The original contributions presented in the study are included in the article/Supplementary Material. Further inquiries can be directed to the corresponding author.

\section{ETHICS STATEMENT}

The animal study was reviewed and approved by NIAID Rocky Mountain Laboratories Animal Care and Use Committee. 


\section{AUTHOR CONTRIBUTIONS}

AM conceived the idea and secured funding. KO'D and AM designed the studies. KO'D, CC, AG, CL, WF, and AM conducted the studies. KO'D, KS, TG, TT, WF, and AM processed the samples and acquired the data. KO'D, CC, and AM analyzed and interpreted the data. $\mathrm{KO} \mathrm{O}^{\prime} \mathrm{D}$, and $\mathrm{AM}$ prepared the manuscript with input from all authors. All authors contributed to the article and approved the submitted version.

\section{FUNDING}

The study was funded by the Intramural Research Program, NIAID, NIH.

\section{REFERENCES}

1. Song F, Shi N, Shan F, Zhang Z, Shen J, Lu H, et al. Emerging 2019 Novel Coronavirus (2019-Ncov) Pneumonia. Radiology (2020) 295(1):210-7. doi: $10.1148 /$ radiol.2020200274

2. World Health Organization. WHO Director-General's Statement on IHR Emergency Committee on Novel Coronavirus (2019-Ncov) [Web Page] (2020). Available at: https://www.who.int/director-general/speeches/detail/ who-director-general-s-statement-on-ihr-emergency-committee-on-novelcoronavirus-(2019-ncov).

3. Guan WJ, Ni ZY, Hu Y, Liang WH, Ou CQ, He JX, et al. Clinical Characteristics of Coronavirus Disease 2019 in China. N Engl J Med (2020) 382(18):1708-20. doi: 10.1056/NEJMoa2002032

4. Hamming I, Timens W, Bulthuis ML, Lely AT, Navis G, van Goor H. Tissue Distribution of ACE2 Protein, the Functional Receptor for SARS Coronavirus. A First Step in Understanding SARS Pathogenesis. J Pathol (2004) 203(2):631-7. doi: 10.1002/path.1570

5. Letko M, Marzi A, Munster V. Functional Assessment of Cell Entry and Receptor Usage for SARS-CoV-2 and Other Lineage B Betacoronaviruses. Nat Microbiol (2020) 5(4):562-9. doi: 10.1038/s41564-020-0688-y

6. Walls AC, Park YJ, Tortorici MA, Wall A, McGuire AT, Veesler D. Structure, Function, and Antigenicity of the SARS-CoV-2 Spike Glycoprotein. Cell (2020) 181(2):281-92 e6. doi: 10.1016/j.cell.2020.02.058

7. Chen N, Zhou M, Dong X, Qu J, Gong F, Han Y, et al. Epidemiological and Clinical Characteristics of 99 Cases of 2019 Novel Coronavirus Pneumonia in Wuhan, China: A Descriptive Study. Lancet (2020) 395(10223):507-13. doi: 10.1016/S0140-6736(20)30211-7

8. Holshue ML, DeBolt C, Lindquist S, Lofy KH, Wiesman J, Bruce H, et al. First Case of 2019 Novel Coronavirus in the United States. N Engl J Med (2020) 382 (10):929-36. doi: 10.1056/NEJMoa2001191

9. Li Q, Guan X, Wu P, Wang X, Zhou L, Tong Y, et al. Early Transmission Dynamics in Wuhan, China, of Novel Coronavirus-Infected Pneumonia. N Engl J Med (2020) 382(13):1199-207. doi: 10.1056/NEJMoa2001316

10. Wu F, Zhao S, Yu B, Chen YM, Wang W, Song ZG, et al. A New Coronavirus Associated With Human Respiratory Disease in China. Nature (2020) 579 (7798):265-9. doi: 10.1038/s41586-020-2008-3

11. Zhou P, Yang XL, Wang XG, Hu B, Zhang L, Zhang W, et al. A Pneumonia Outbreak Associated With a New Coronavirus of Probable Bat Origin. Nature (2020) 579(7798):270-3. doi: 10.1038/s41586-020-2012-7

12. Rambaut A, Loman N, Pybus O, Barclay W, Barrett J, Carabelli6 A, et al. Preliminary Genomic Characterisation of an Emergent SARS-CoV-2 Lineage in the UK Defined by a Novel Set of Spike Mutations 2020. Available at: https://virological.org/t/preliminary-genomic-characterisation-of-anemergent-sars-cov-2-lineage-in-the-uk-defined-by-a-novel-set-of-spikemutations/563.

13. Faria NR, Mellan TA, Whittaker C, Claro IM, Candido D, Mishra S, et al. Genomics and Epidemiology of the P.1 SARS-CoV-2 Lineage in Manaus, Brazil. Science (2021) 372(6544):815-21. doi: 10.1126/science.abh2644

\section{ACKNOWLEDGMENTS}

The authors thank the Rocky Mountain Veterinary Branch, NIAID for supporting the animal studies, and Anita Mora (NIAID) for assistance generating the pathology figures. The authors also thank members of the Molecular Pathogenesis Unit, Virus Ecology Section, and Research Technology Branch (all NIAID) for their efforts to obtain and characterize the SARS-CoV-2 isolates.

\section{SUPPLEMENTARY MATERIAL}

The Supplementary Material for this article can be found online at: https://www.frontiersin.org/articles/10.3389/fimmu.2021.788235/ full\#supplementary-material

14. Chen RE, Zhang X, Case JB, Winkler ES, Liu Y, VanBlargan LA, et al. Resistance of SARS-CoV-2 Variants to Neutralization by Monoclonal and Serum-Derived Polyclonal Antibodies. Nat Med (2021) 27(4):717-26. doi: 10.1038/s41591-021-01294-w

15. Liu Y, Liu J, Xia H, Zhang X, Fontes-Garfias CR, Swanson KA, et al. Neutralizing Activity of BNT162b2-Elicited Serum. N Engl J Med (2021) 384(15):1466-8. doi: 10.1056/NEJMc2102017

16. Wibmer CK, Ayres F, Hermanus T, Madzivhandila M, Kgagudi P, Oosthuysen B, et al. SARS-CoV-2 501y.V2 Escapes Neutralization by South African COVID-19 Donor Plasma. Nat Med (2021) 27(4):622-5. doi: 10.1038/s41591-021-01285-x

17. US Food and Drug Administration. FDA Approves First COVID-19 Vaccine [Press Release]. Available at: https://www.fda.gov/news-events/pressannouncements/fda-approves-first-covid-19-vaccine2021.

18. Corbett KS, Flynn B, Foulds KE, Francica JR, Boyoglu-Barnum S, Werner AP, et al. Evaluation of the mRNA-1273 Vaccine Against SARS-CoV-2 in Nonhuman Primates. N Engl J Med (2020) 383(16):1544-55. doi: 10.1056/ NEJMoa2024671

19. Vogel AB, Kanevsky I, Che Y, Swanson KA, Muik A, Vormehr M, et al. (2020).

20. Sadoff J, Le Gars M, Shukarev G, Heerwegh D, Truyers C, de Groot AM, et al. Interim Results of a Phase 1-2a Trial of Ad26.COV2.S Covid-19 Vaccine. $N$ Engl J Med (2021) 384(19):1824-35. doi: 10.1056/NEJMoa2034201

21. Hassan AO, Kafai NM, Dmitriev IP, Fox JM, Smith BK, Harvey IB, et al. A Single-Dose Intranasal ChAd Vaccine Protects Upper and Lower Respiratory Tracts Against SARS-CoV-2. Cell (2020) 183(1):169-84 e13. doi: 10.1016/ j.cell.2020.08.026

22. An X, Martinez-Paniagua M, Rezvan A, Fathi M, Singh S, Biswas S, et al. SingleDose Intranasal Vaccination Elicits Systemic and Mucosal Immunity Against SARS-CoV-2. iScience (2021) 24(9):103037. doi: 10.1101/2020.07.23.212357

23. van Doremalen N, Purushotham JN, Schulz JE, Holbrook MG, Bushmaker T, Carmody A, et al. Intranasal ChAdOx1 Ncov-19/AZD1222 Vaccination Reduces Viral Shedding After SARS-CoV-2 D614G Challenge in Preclinical Models. Sci Trans Med (2021) 13(607):eabh0755. doi: 10.1126/scitranslmed. abh0755

24. King RG, Silva-Sanchez A, Peel JN, Botta D, Dickson AM, Pinto AK, et al. Single-Dose Intranasal Administration of AdCOVID Elicits Systemic and Mucosal Immunity Against SARS-CoV-2 and Fully Protects Mice From Lethal Challenge. Vaccines (Basel) (2021) 9(8). doi: 10.3390/vaccines9080881

25. Safronetz D, Mire C, Rosenke K, Feldmann F, Haddock E, Geisbert T, et al. A Recombinant Vesicular Stomatitis Virus-Based Lassa Fever Vaccine Protects Guinea Pigs and Macaques Against Challenge With Geographically and Genetically Distinct Lassa Viruses. PloS Negl Trop Dis (2015) 9(4):e0003736. doi: 10.1371/journal.pntd.0003736

26. Mire CE, Geisbert JB, Agans KN, Versteeg KM, Deer DJ, Satterfield BA, et al. Use of Single-Injection Recombinant Vesicular Stomatitis Virus Vaccine to Protect Nonhuman Primates Against Lethal Nipah Virus Disease. Emerg Infect Dis (2019) 25(6):1144-52. doi: 10.3201/eid2506.181620 
27. Marzi A, Feldmann H, Geisbert TW, Falzarano D. Vesicular Stomatitis VirusBased Vaccines for Prophylaxis and Treatment of Filovirus Infections. J Bioterror Biodef (2011) S1(4):2157-2526-S1-004. doi: 10.4172/21572526.S1-004

28. Dieterle ME, Haslwanter D, Bortz RH3rd, Wirchnianski AS, Lasso G, Vergnolle O, et al. A Replication-Competent Vesicular Stomatitis Virus for Studies of SARS-CoV-2 Spike-Mediated Cell Entry and Its Inhibition. Cell Host Microbe (2020) 28(3):486-96 e6. doi: 10.1016/j.chom.2020.06.020

29. Case JB, Rothlauf PW, Chen RE, Kafai NM, Fox JM, Smith BK, et al. Replication-Competent Vesicular Stomatitis Virus Vaccine Vector Protects Against SARS-CoV-2-Mediated Pathogenesis in Mice. Cell Host Microbe (2020) 28(3):465-74 e4. doi: 10.1016/j.chom.2020.07.018

30. Yahalom-Ronen Y, Tamir H, Melamed S, Politi B, Shifman O, Achdout H, et al. A Single Dose of Recombinant VSV-G-Spike Vaccine Provides Protection Against SARS-CoV-2 Challenge. Nat Commun (2020) 11 (1):6402. doi: 10.1038/s41467-020-20228-7

31. Fathi A, Dahlke C, Addo MM. Recombinant Vesicular Stomatitis Virus Vector Vaccines for WHO Blueprint Priority Pathogens. Hum Vaccin Immunother (2019) 15(10):2269-85. doi: 10.1080/21645515.2019.1649532

32. Marzi A, Robertson SJ, Haddock E, Feldmann F, Hanley PW, Scott DP, et al. EBOLA VACCINE. VSV-EBOV Rapidly Protects Macaques Against Infection With the 2014/15 Ebola Virus Outbreak Strain. Science (2015) 349(6249):73942. doi: 10.1126/science.aab3920

33. Brown KS, Safronetz D, Marzi A, Ebihara H, Feldmann H. Vesicular Stomatitis Virus-Based Vaccine Protects Hamsters Against Lethal Challenge With Andes Virus. J Virol (2011) 85(23):12781-91. doi: 10.1128/JVI.00794-11

34. Furuyama W, Reynolds P, Haddock E, Meade-White K, Quynh Le M, Kawaoka Y, et al. A Single Dose of a Vesicular Stomatitis Virus-Based Influenza Vaccine Confers Rapid Protection Against H5 Viruses From Different Clades. NPJ Vaccines (2020) 5(1). doi: 10.1038/s41541-019-0155-Z

35. Henao-Restrepo AM, Camacho A, Longini IM, Watson CH, Edmunds WJ, Egger M, et al. Efficacy and Effectiveness of an rVSV-Vectored Vaccine in Preventing Ebola Virus Disease: Final Results From the Guinea Ring Vaccination, Open-Label, Cluster-Randomised Trial (Ebola Ça Suffit)! Lancet (2017) 389(10068):505-18. doi: 10.1016/S0140-6736(16)32621-6

36. Furuyama W, Shifflett K, Pinksi AN, Griffin AJ, Feldmann F, Okumura A, et al. Rapid Protection From COVID-19 in Nonhuman Primates Vaccinated Intramuscularly But Not Intranasally With a Single Dose of a Recombinant Vaccine. bioRxiv (2021). doi: 10.1101/2021.01.19.426885

37. Imai M, Iwatsuki-Horimoto K, Hatta M, Loeber S, Halfmann PJ, Nakajima N, et al. Syrian Hamsters as a Small Animal Model for SARS-CoV-2 Infection and Countermeasure Development. Proc Natl Acad Sci USA (2020) 117 (28):16587-95. doi: 10.1073/pnas.2009799117

38. Rosenke K, Meade-White K, Letko M, Clancy C, Hansen F, Liu Y, et al. Defining the Syrian Hamster as a Highly Susceptible Preclinical Model for SARS-CoV-2 Infection. Emerg Microbes Infect (2020) 9(1):2673-84. doi: $10.1080 / 22221751.2020 .1858177$

39. Takamura S, Yagi H, Hakata Y, Motozono C, McMaster SR, Masumoto T, et al. Specific Niches for Lung-Resident Memory CD8+ T Cells at the Site of Tissue Regeneration Enable CD69-Independent Maintenance. J Exp Med (2016) 213(13):3057-73. doi: 10.1084/jem.20160938

40. Harcourt J, Tamin A, Lu X, Kamili S, Sakthivel SK, Murray J, et al. Severe Acute Respiratory Syndrome Coronavirus 2 From Patient With Coronavirus Disease, United States. Emerg Infect Dis (2020) 26(6):1266-73. doi: 10.3201/ eid2606.200516

41. Tsuda Y, Safronetz D, Brown K, LaCasse R, Marzi A, Ebihara H, et al. Protective Efficacy of a Bivalent Recombinant Vesicular Stomatitis Virus Vaccine in the Syrian Hamster Model of Lethal Ebola Virus Infection. J Infect Dis (2011) 204 Suppl 3:S1090-7. doi: 10.1093/infdis/jir379

42. Emanuel J, Callison J, Dowd KA, Pierson TC, Feldmann H, Marzi A. A VSVBased Zika Virus Vaccine Protects Mice From Lethal Challenge. Sci Rep (2018) 8(1):11043. doi: 10.1038/s41598-018-29401-x

43. van Doremalen N, Lambe T, Spencer A, Belij-Rammerstorfer S, Purushotham JN, Port JR, et al. ChAdOx1 nCoV-19 Vaccine Prevents SARS-CoV-2 Pneumonia in Rhesus Macaques. Nature (2020) 586(7830):578-82. doi: 10.1038/s41586-020-2608-y

44. Barrigan LM, Tuladhar S, Brunton JC, Woolard MD, Chen CJ, Saini D, et al. Infection With Francisella Tularensis LVS clpB Leads to an Altered Yet
Protective Immune Response. Infect Immun (2013) 81(6):2028-42. doi: 10.1128/IAI.00207-13

45. Case JB, Rothlauf PW, Chen RE, Liu Z, Zhao H, Kim AS, et al. Neutralizing Antibody and Soluble ACE2 Inhibition of a Replication-Competent VSVSARS-CoV-2 and a Clinical Isolate of SARS-CoV-2. Cell Host Microbe (2020) 28(3):475-85.e5. doi: 10.1016/j.chom.2020.06.021

46. Centers for Disease Control and Prevention. Emerging SARS-CoV-2 Variants 2021. Available at: https://www.cdc.gov/coronavirus/2019-ncov/more/ science-and-research/scientific-brief-emerging-variants.html.

47. Channappanavar R, Fett C, Zhao J, Meyerholz DK, Perlman S. Virus-Specific Memory CD8 T Cells Provide Substantial Protection From Lethal Severe Acute Respiratory Syndrome Coronavirus Infection. J Virol (2014) 88 (19):11034-44. doi: 10.1128/JVI.01505-14

48. Channappanavar R, Zhao J, Perlman S. T Cell-Mediated Immune Response to Respiratory Coronaviruses. Immunol Res (2014) 59(1-3):118-28. doi: 10.1007/ s12026-014-8534-Z

49. Zhao J, Zhao J, Perlman S. T Cell Responses are Required for Protection From Clinical Disease and for Virus Clearance in Severe Acute Respiratory Syndrome Coronavirus-Infected Mice. J Virol (2010) 84(18):9318-25. doi: 10.1128/JVI.01049-10

50. Thanh Le T, Andreadakis Z, Kumar A, Gomez Roman R, Tollefsen S, Saville M, et al. The COVID-19 Vaccine Development Landscape. Nat Rev Drug Discovery (2020) 19(5):305-6. doi: 10.1038/d41573-020-00073-5

51. Merck (News Release). Merck Discontinues Development of SARS-CoV-2/ COVID-19 Vaccine Candidates; Continues Development of Two Investigational Therapeutic Candidates (2021). Available at: https://www.merck.com/news/ merck-discontinues-development-of-sars-cov-2-covid-19-vaccine-candidatescontinues-development-of-two-investigational-therapeutic-candidates/.

52. Sungnak W, Huang N, Becavin C, Berg M, Queen R, Litvinukova M, et al. SARS-CoV-2 Entry Factors are Highly Expressed in Nasal Epithelial Cells Together With Innate Immune Genes. Nat Med (2020) 26(5):681-7. doi: 10.1038/s41591-020-0868-6

53. O’Donnell KL, Pinski AN, Clancy CS, Gourdine T, Shifflett K, Fletcher P, et al. Pathogenic and Transcriptomic Differences of Emerging SARS-CoV-2 Variants in the Syrian Golden Hamster Model. EBioMedicine (2021) 73:103675. doi: 10.1016/j.ebiom.2021.103675

54. Garcia-Beltran WF, Lam EC, St Denis K, Nitido AD, Garcia ZH, Hauser BM, et al. Multiple SARS-CoV-2 Variants Escape Neutralization by VaccineInduced Humoral Immunity. Cell (2021) 184(9):2372-83.e9. doi: 10.1016/ j.cell.2021.03.013

55. Noori M, Nejadghaderi SA, Arshi S, Carson-Chahhoud K, Ansarin K, Kolahi AA, et al. Potency of BNT162b2 and mRNA-1273 Vaccine-Induced Neutralizing Antibodies Against Severe Acute Respiratory Syndrome-CoV-2 Variants of Concern: A Systematic Review of In Vitro Studies. Rev Med Virol (2021) e2277. doi: 10.1002/rmv.2277

56. Knoll MD, Wonodi C. Oxford-AstraZeneca COVID-19 Vaccine Efficacy. Lancet (2021) 397(10269):72-4. doi: 10.1016/S0140-6736(20)32623-4

57. Harvey WT, Carabelli AM, Jackson B, Gupta RK, Thomson EC, Harrison EM, et al. SARS-CoV-2 Variants, Spike Mutations and Immune Escape. Nat Rev Microbiol (2021) 19(7):409-24. doi: 10.1038/s41579-021-00573-0

58. Madhi SA, Baillie V, Cutland CL, Voysey M, Koen AL, Fairlie L, et al. Efficacy of the ChAdOx1 Ncov-19 Covid-19 Vaccine Against the B.1.351 Variant. N Engl J Med (2021) 384(20):1885-98. doi: 10.1056/NEJMoa2102214

59. Fischer RJ, van Doremalen N, Adney DR, Yinda CK, Port JR, Holbrook MG, et al. ChAdOx1 nCoV-19 (AZD1222) Protects Hamsters Against SARS-CoV2 B.1.351 and B.1.1.7 Disease. Nat Commun (2021) 12(1):5868. doi: 10.1038/ s41467-021-26178-y

60. Wuertz KM, Barkei EK, Chen WH, Martinez EJ, Lakhal-Naouar I, Jagodzinski LL, et al. A SARS-CoV-2 Spike Ferritin Nanoparticle Vaccine Protects Against Heterologous Challenge With B.1.1.7 and B.1.351 Virus Variants in Syrian Golden Hamsters. bioRxiv (2021). doi: 10.1101/ 2021.06.16.448525

61. Fisher E, Padula L, Podack K, O’Neill K, Seavey MM, Jayaraman P, et al. Induction of SARS-CoV-2 Protein S-Specific CD8+ T Cells in the Lungs of Gp96-Ig-S Vaccinated Mice. Front Immunol (2020) 11:602254. doi: 10.3389/ fimmu.2020.602254

62. Shinoda K, Tokoyoda K, Hanazawa A, Hayashizaki K, Zehentmeier S, Hosokawa H, et al. Type II Membrane Protein CD69 Regulates the 
Formation of Resting T-Helper Memory. Proc Natl Acad Sci USA (2012) 109 (19):7409-14. doi: 10.1073/pnas.1118539109

63. Kirby T. New Variant of SARS-CoV-2 in UK Causes Surge of COVID-19. Lancet Respir Med (2021) 9(2):e20-21. doi: 10.1016/S2213-2600(21)00005-9

Conflict of Interest: The authors declare that the research was conducted in the absence of any commercial or financial relationships that could be construed as a potential conflict of interest.

Publisher's Note: All claims expressed in this article are solely those of the authors and do not necessarily represent those of their affiliated organizations, or those of the publisher, the editors and the reviewers. Any product that may be evaluated in this article, or claim that may be made by its manufacturer, is not guaranteed or endorsed by the publisher.

Copyright (๔) 2022 O'Donnell, Clancy, Griffin, Shifflett, Gourdine, Thomas, Long, Furuyama and Marzi. This is an open-access article distributed under the terms of the Creative Commons Attribution License (CC BY). The use, distribution or reproduction in other forums is permitted, provided the original author(s) and the copyright owner(s) are credited and that the original publication in this journal is cited, in accordance with accepted academic practice. No use, distribution or reproduction is permitted which does not comply with these terms. 